\title{
A Tangent Linear Approximation of the Ignition Delay Time. II: Sensitivity to Thermochemical Parameters
}

\author{
Mireille Hantouche $^{\mathrm{a}}$, Saja Almohammadi ${ }^{\mathrm{b}}$, Olivier P. Le Maître ${ }^{\mathrm{c}}$, Omar M. Knio ${ }^{\mathrm{b}, *}$ \\ ${ }^{a}$ Division of Physical Science and Engineering, King Abdullah University of Science and Technology, Thuwal, Saudi Arabia \\ ${ }^{b}$ Division of Computer, Electrical, and Mathematical Sciences and Engineering, King Abdullah University of Science and \\ Technology, Thuwal, Saudi Arabia \\ ${ }^{c}$ CNRS, Inria, Centre de Mathématiques Appliquées, Ecole Polytechnique, 91120 Palaiseau, France
}

\begin{abstract}
The tangent linear approximation (TLA) developed in Almohammadi et al. (Combust. Flame 230, 111426) is extended to estimate the sensitivity of the ignition delay time with respect to species enthalpies and entropies. The proposed method relies on integrating the linearized system of equations governing the evolution of the state vector's partial derivatives with respect to variations in thermodynamic parameters. The sensitivity of the ignition delay time is estimated through a linearized approximation of a temperature functional. The TLA approach is applied to three gas mixtures, $\mathrm{H}_{2}$, n-butanol, and iso-octane, reacting in air under adiabatic, constant-volume conditions. The numerical experiments indicate that the linearized approximation of the ignition delay time's sensitivity is in excellent agreement with the finite-difference estimates. This is also the case for sensitivity estimates obtained using the TLA approach. Further, significant computational speed-ups are achieved with the TLA approach, and the method scales well with the number of perturbed parameters. In the case of the $\mathrm{H}_{2}$ mechanism, TLA is about ten times faster than finite differences, and this enhancement becomes even more substantial when more complex mechanisms are considered.
\end{abstract}

Keywords: local sensitivity; tangent linear approximation; thermophysical parameter; Jacobian

\section{Introduction}

Sensitivity analyses are frequently employed to identify the key parameters contributing to the variability in quantities of interest (QoIs) predicted by a chemical reaction model. In particular, these methods have been applied to guide model reduction and refinement. A prominent example of the application of sensitivity tools in the context of ignition simulations concerns the characterization of the ignition delay time $\left(\tau_{\text {ign }}\right.$, defined precisely later) to local perturbations in chemical rate parameters.

In contrast to the extensive body of work focusing on the impact of rate parameters, a relatively lower number of studies have addressed the role of thermodynamic properties on mixture reactivity [1-8]. This is a primary focus of the present work.

Traditionally, sensitivity analyses have relied on a (so-called brute-force) finite-difference methodology based on perturbing one-at-a-time the input parameters and performing independent simulations of the system to determine the perturbed QoI. When $N$ independent parameters are considered, this requires $N+1$ independent simulations if first-order differences are used, or $2 N+1$ in the case of centered differences.

Recently, however, several approaches [9-12] have been conducted that focus on exploring alternative means of estimating sensitivities that can specifically reduce the $\mathrm{O}(N)$ cost associated with the brute force approach. These include methodologies based on tangent linear approximations [9, 12-14], adjoint techniques [10], as well as optimization approaches [11]. Applications in [9-12] have particularly focused on

*Corresponding author. Email: omar.knio@kaust.edu.sa 
estimating ignition delay time sensitivities to rate parameters. These works have shown that the underlying techniques offer accurate and efficient means of estimating sensitivities and that, in high dimensional situations, speedup by order-of-magnitude can be achieved over the traditional brute-force finite-difference approach.

In this work, we focus on extending the TLA methodology introduced in our previous work [12] to enable estimating the local sensitivity of the ignition delay time to variations in the thermodynamic properties of individual species. Note that regardless of the parameters being perturbed, the TLA methodology leads to a system of dynamical equations that governs the state-vector perturbations [9-15], comprising a linear stretching term involving the Jacobian of the source term, and a forcing term that corresponds to the derivative of the source term with respect to the parameters being perturbed. Our effort in [12] focused exclusively on the impact of rate parameters, and consequently involved deriving expressions for the derivative of the source term with respect to these parameters. In section 2 , a similar analysis is applied, focusing on enthalpy and entropy perturbations, assuming both to be temperature independent. As in [12], we rely on a linearized functional approximation to relate the sensitivity of the ignition delay time to the sensitivity of the state vector. In Section 3, a unified framework is presented that enables simultaneous characterization of the impact of reaction rate, enthalpy, and entropy perturbations. Section 4 provides a brief description of the simulation approaches for modeling the ignition problem and simulating the evolution of local sensitivities. The application of the TLA framework presently developed is illustrated in section 5 for simulations of the ignition of hydrogen/air, iso-octane/air, and n-butanol/air mixtures. The main conclusions of the work are discussed in section 6 .

\section{Formulation}

\subsection{Governing Equations}

In this section, we start by summarizing the governing equation for homogeneous combustion of a reacting gas mixture under adiabatic, constant-volume conditions; see Refs. [16, 17] and Appendix A. We denote $Y_{i}, i=1, \ldots, N_{s}$ the species mass fractions, $N_{s}$ the number of reacting species, $T$ the temperature, and $t$ the time. For a detailed chemical mechanism involving $N_{r}$ elementary reactions, the evolution of the species mass fractions and temperature follows the coupled system,

$$
\begin{aligned}
\frac{d Y_{i}}{d t} & =\frac{1}{\rho} \sum_{j=1}^{N_{r}}\left[\dot{f}_{i}^{j}+\dot{r}_{i}^{j}\right], \quad i=1, \ldots, N_{s} \\
\frac{d T}{d t} & =-\frac{\gamma}{\rho c_{p}} \sum_{i=1}^{N_{s}} h_{i}\left(\sum_{j=1}^{N_{r}}\left[\dot{f}_{i}^{j}+\dot{r}_{i}^{j}\right]\right)+(\gamma-1) \frac{T W}{\rho} \sum_{i=1}^{N_{s}} \frac{1}{W_{i}}\left(\sum_{j=1}^{N_{r}}\left[\dot{f}_{i}^{j}+\dot{r}_{i}^{j}\right]\right),
\end{aligned}
$$

where $\rho$ denotes the density, $c_{p}$ is the heat capacity at constant pressure, $\dot{f}_{i}^{j}$ is forward rate of production of species $i$ due to reaction $j, \dot{r}_{i}^{j}$ is the reverse rate of production of species $i$ due to reaction $j$, $\gamma$ is the specific heat ratio, $h_{i}$ is the enthalpy of species $i, W_{i}$ is the molecular weight of species $i$, whereas

$$
W \equiv\left(\sum_{i=1}^{N_{s}} \frac{Y_{i}}{W_{i}}\right)^{-1}
$$

The species forward and reverse rates, respectively $\dot{f}_{i}^{j}$ and $\dot{r}_{i}^{j}$, are defined such the total reaction rate of species $i, \dot{\omega}_{i}$, is given by $\dot{\omega}_{i}=\sum_{j=1}^{N_{r}}\left[\dot{f}_{i}^{j}+\dot{r}_{i}^{j}\right]$.

\subsection{Setup}

Our main objective is to estimate the sensitivity of the ignition delay time, $\tau_{\text {ign }}$, with respect to infinitesimal perturbations in the stochastic germ parametrizing the uncertainty in species enthalpies and 
entropies $[2-4,8]$. The latter are typically expressed using NASA polynomials, according to $[17,18]$ :

$$
\begin{aligned}
\frac{H_{i}}{\Re} & =T\left(a_{0, i}+T\left(\frac{a_{1, i}}{2}+T\left(\frac{a_{2, i}}{3}+T\left(\frac{a_{3, i}}{4}+\frac{a_{4, i}}{5} T\right)\right)\right)\right)+a_{5, i}, \\
\frac{S_{i}}{\Re} & =a_{0, i} \ln T+T\left(a_{1, i}+T\left(\frac{a_{2, i}}{2}+T\left(\frac{a_{3, i}}{3}+\frac{a_{4, i}}{4} T\right)\right)\right)+a_{6, i},
\end{aligned}
$$

where $H_{i}$ and $S_{i}$ are the molar enthalpies, and $\Re$ is the universal gas constant. The specific thermodynamic properties in mass units are obtained by dividing the above expressions by the molecular weights,

$$
h_{i}=H_{i} / W_{i}, \quad s_{i}=S_{i} / W_{i} .
$$

Following [8], with the molar enthalpy and entropy of species $i$ respectively represented as in (3) and (4), we assume that parameters $a_{5, i}$ and $a_{6, i}$ are uncertain and parametrized using independent canonical random variables $\eta_{i}$ and $\sigma_{i}$, uniformly distributed over $[-1,1]$. The dependence of $a_{5, i}$ and $a_{6, i}$ on $\eta_{i}$ and $\sigma_{i}$ is expressed as

$$
a_{5, i}\left(\eta_{i}\right)=a_{5, i}^{0}+\alpha_{i} \eta_{i}, \quad a_{6, i}\left(\sigma_{i}\right)=a_{6, i}^{0}+\beta_{i} \sigma_{i},
$$

where $a_{5, i}^{0}$ and $a_{6, i}^{0}$ denote the respective nominal values, whereas $\alpha_{i}$ and $\beta_{i}$ denote the corresponding (prior) ranges. Clearly, we have:

$$
\frac{\partial h_{i}}{\partial \eta_{k}}=\frac{\alpha_{i} \Re}{W_{i}} \delta_{i k}, \quad \frac{\partial h_{i}}{\partial \sigma_{l}}=0, \quad \frac{\partial s_{i}}{\partial \eta_{k}}=0, \quad \frac{\partial s_{i}}{\partial \sigma_{l}}=\frac{\beta_{i} \Re}{W_{i}} \delta_{i l},
$$

where $\delta$ denotes the Kronecker delta.

In the present setup, our goal is to develop an efficient methodology that enables us to estimate the local sensitivity of the ignition delay time with respect to infinitesimal perturbations of individual canonical variables $\eta_{i}$ and $\sigma_{i}$, i.e. to determine the partial derivatives $\partial \tau_{\mathrm{ign}} / \partial \eta_{i}$, and $\partial \tau_{\mathrm{ign}} / \partial \sigma_{i}$, as well as the local sensitivity indices defined as

$$
\mathrm{S}_{i}^{\mathrm{h}} \equiv h_{i}\left(\frac{\partial h_{i}}{\partial \eta_{i}}\right)^{-1} \frac{1}{\tau_{\mathrm{ign}}} \frac{\partial \tau_{\mathrm{ign}}}{\partial \eta_{i}}, \quad \text { and } \quad \mathrm{S}_{i}^{\mathrm{S}} \equiv s_{i}\left(\frac{\partial s_{i}}{\partial \sigma_{i}}\right)^{-1} \frac{1}{\tau_{\mathrm{ign}}} \frac{\partial \tau_{\mathrm{ign}}}{\partial \sigma_{i}} .
$$

\subsection{Dependence of reaction rates on selected thermophysical parameters}

When both the forward and reverse reaction rates are specified using Arrhenius parameters, variations of species enthalpies and entropies do not impact the reaction rates. On the other hand, when reverse Arrhenius parameters are not specified, the reverse rate parameter, $k_{r j}$, is computed based on the forward rate $k_{f_{j}}$, and the equilibrium constant, $K_{c j}$, namely according to [17],

$$
k_{r j}=\frac{k_{f j}}{K_{c j}}
$$

where

$$
K_{c j}=\left(\frac{p_{a t m}}{\Re}\right)^{\sum_{k=1}^{N_{s}} \nu_{k j}} \exp \left(\sum_{k=1}^{N_{s}} \nu_{k j} g_{k}\right),
$$

$\nu_{k j}$ is the stoichiometric coefficient of species $k$ in reaction $j$, and

$$
g_{k}=a_{6, k}-a_{0, k}+\left(a_{0, k}-1\right) \ln T+T\left(\frac{a_{1, k}}{2}+T\left(\frac{a_{2, k}}{6}+T\left(\frac{a_{3, k}}{12}+\frac{a_{4, k}}{20} T\right)\right)\right)-\frac{a_{5, k}}{T} .
$$

Differentiating (8), and using (9-10), it follows that

$$
\frac{\partial k_{r j}}{\partial \eta_{l}}=-k_{r j} \frac{\partial}{\partial \eta_{l}}\left(\sum_{k=1}^{N_{s}} \nu_{k j} g_{k}\right)=k_{r j} \frac{\nu_{l j} \alpha_{l}}{T} .
$$

Similarly, we have

$$
\frac{\partial k_{r j}}{\partial \sigma_{l}}=-k_{r j} \nu_{l j} \beta_{l}
$$




\subsection{Tangent linear approximation}

To estimate the ignition delay time's sensitivity for infinitesimal perturbations in thermophysical parameters, we rely on linearized approximations of the governing equation. For variations in enthalpies, the linearization is performed with respect to local variations in the $\eta_{k}$, whereas when entropy variations are considered, the linearization is performed for variations in the $\sigma_{k}$.

Let $\boldsymbol{Z}(t)$ denote the state vector concatenating the species mass fractions and temperature for a given realization of the germ:

$$
Z_{i}= \begin{cases}Y_{i} & i=1, \ldots, N_{s} \\ T & i=N_{s+1}\end{cases}
$$

Following a similar methodology as in $[12,13]$, the local sensitivity of the state around $\boldsymbol{Z}(t)$, with respect to enthalpy variations $\eta_{k=1, \ldots, N_{s}}$, is estimated by integrating the linearized system

$$
\frac{d \boldsymbol{Z}_{, k}^{\mathrm{h}}}{d t}=\mathbf{J}(t) \boldsymbol{Z}_{, k}^{\mathrm{h}}+\boldsymbol{S}_{k}^{\eta} \quad k=1, \ldots, N_{s},
$$

from the initial condition $\boldsymbol{Z}_{, k}^{\mathrm{h}}(0)=0$. Here $\boldsymbol{Z}_{, k}^{\mathrm{h}}(t) \equiv \partial \boldsymbol{Z} / \partial \eta_{k}$ denotes the partial derivative of the state at $\boldsymbol{Z}(t), \mathbf{J}(t)$ is the Jacobian of the right-hand side of (1) at $\boldsymbol{Z}(t), \boldsymbol{S}_{k}^{\eta}$ is the vector with components $S_{i k}^{\eta}$ given by

$$
S_{i k}^{\eta}(t)=\frac{1}{\rho} \sum_{j=1}^{N_{r}} q_{j k}^{\eta} \dot{r}_{i}^{j}, \quad i=1, \ldots, N_{s},
$$

and

$$
S_{N_{s}+1, k}^{\eta}=-\frac{\gamma}{\rho c_{p}} \frac{\alpha_{k} \Re}{W_{k}}\left(\sum_{j=1}^{N_{r}} \dot{\omega}_{k}^{j}\right)-\frac{\gamma}{\rho c_{p}} \sum_{i=1}^{N_{s}} h_{i}\left(\sum_{j=1}^{N_{r}} q_{j k}^{\eta} \dot{r}_{i}^{j}\right)+(\gamma-1) \frac{T W}{\rho} \sum_{i=1}^{N_{s}} \frac{1}{W_{i}}\left(\sum_{j=1}^{N_{r}} q_{j k}^{\eta} \dot{r}_{i}^{j}\right),
$$

where $\dot{\omega}_{i}^{j} \equiv \dot{f}_{i}^{j}+\dot{r}_{i}^{j}$, and

$$
q_{j k}^{\eta} \equiv I_{j} \frac{\nu_{k j} \alpha_{k}}{T}, \quad 1 \leq k \leq N_{s}, \quad 1 \leq j \leq N_{r} .
$$

The reaction indicator, $I_{j}$, is defined according to

$$
I_{j}= \begin{cases}0 & \text { reverse Arrhenius parameters for reaction } j \text { are specified } \\ 1 & \text { otherwise. }\end{cases}
$$

We use a similar approach to estimate the sensitivity of the state with respect to variations in $\sigma_{l=1, \ldots, N_{s}}$. In that case, the variations are governed by

$$
\frac{d \boldsymbol{Z}_{, l}^{\mathrm{s}}}{d t}=\mathbf{J}(t) \boldsymbol{Z}_{, l}^{\mathrm{s}}+\boldsymbol{S}_{l}^{\sigma} \quad l=1, \ldots, N_{s},
$$

with initial condition $\boldsymbol{Z}_{l,}^{\mathrm{s}}(0)=0$, whereas $\boldsymbol{Z}_{l,}^{\mathrm{s}}(t) \equiv \partial \boldsymbol{Z} / \partial \sigma_{l}$ denotes the partial derivative of the state at $\boldsymbol{Z}(t)$, and $\mathbf{J}(t)$ is again the Jacobian of the right-hand side of (1) at $\boldsymbol{Z}(t)$. The source term $\boldsymbol{S}_{l}^{\sigma}$ in (19) is given component-wise by:

$$
S_{i l}^{\sigma}(t)= \begin{cases}-\frac{1}{\rho} \sum_{j=1}^{N_{r}} q_{j l}^{\sigma} \dot{r}_{i}^{j}, & i=1, \ldots, N_{s}, \\ \frac{\gamma}{\rho c_{p}} \sum_{i=1}^{N_{s}} h_{i}\left(\sum_{j=1}^{N_{r}} q_{j l}^{\sigma} \dot{r}_{i}^{j}\right)-(\gamma-1) \frac{T W}{\rho} \sum_{i=1}^{N_{s}} \frac{1}{W_{i}}\left(\sum_{j=1}^{N_{r}} q_{j l}^{\sigma} \dot{r}_{i}^{j}\right) & i=N_{s}+1,\end{cases}
$$

where

$$
q_{j l}^{\sigma} \equiv I_{j} \nu_{l j} \beta_{l}, \quad 1 \leq j \leq N_{r}, \quad 1 \leq l \leq N_{s} .
$$




\subsection{Canonical Systems}

Note that because of the linearity of the governing equations (14) and (19), and the proportionality of the source terms with $\alpha_{k}$ and $\sigma_{l}$ respectively, it is convenient to define canonical systems in which the enthalpies and entropies have equal uncertainty ranges. We denote by $\hat{\alpha}$ and $\hat{\beta}$ the canonical values of the ranges for enthalpy and entropy respectively, to distinguish them from the actual values $\alpha_{k}$ and $\beta_{l}$ associated with individual species. Using the canonical values, we may consider the state vectors $\hat{Z}_{, k}^{\mathrm{h}}$ and $\hat{\boldsymbol{Z}}_{, l}^{\mathrm{s}}$, respectively governed by

$$
\frac{d \hat{\boldsymbol{Z}}_{, k}^{\mathrm{h}}}{d t}=\mathbf{J}(t) \hat{\boldsymbol{Z}}_{, k}^{\mathrm{h}}+\hat{\boldsymbol{S}}_{k}^{\eta}, \quad \frac{d \hat{\boldsymbol{Z}}_{, l}^{\mathrm{s}}}{d t}=\mathbf{J}(t) \hat{\boldsymbol{Z}}_{, l}^{\mathrm{s}}+\hat{\boldsymbol{S}}_{l}^{\sigma}
$$

for $k=1, \ldots, N_{s}$ and $l=1, \ldots, N_{s}$, with

$$
\hat{S}_{i k}^{\eta}(t)=\frac{1}{\rho} \sum_{j=1}^{N_{r}} \hat{q}_{j k}^{\eta} \dot{r}_{i}^{j}, \quad \hat{S}_{i l}^{\sigma}(t)=-\frac{1}{\rho} \sum_{j=1}^{N_{r}} \hat{q}_{j l}^{\sigma} \dot{r}_{i}^{j}, \quad i=1, \ldots, N_{s},
$$

and

$$
\hat{S}_{N_{s}+1, k}^{\eta}=-\frac{\gamma}{\rho c_{p}} \frac{\hat{\alpha} \Re}{W_{k}}\left(\sum_{j=1}^{N_{r}} \dot{\omega}_{k}^{j}\right)-\frac{\gamma}{\rho c_{p}} \sum_{i=1}^{N_{s}} h_{i}\left(\sum_{j=1}^{N_{r}} \hat{q}_{j k}^{\eta} \dot{r}_{i}^{j}\right)+(\gamma-1) \frac{T W}{\rho} \sum_{i=1}^{N_{s}} \frac{1}{W_{i}}\left(\sum_{j=1}^{N_{r}} \hat{q}_{j k}^{\eta} \dot{r}_{i}^{j}\right),
$$

where

$$
\hat{S}_{N_{s}+1, l}^{\sigma}(t)=\frac{\gamma}{\rho c_{p}} \sum_{i=1}^{N_{s}} h_{i}\left(\sum_{j=1}^{N_{r}} \hat{q}_{j l}^{\sigma} \dot{r}_{i}^{j}\right)-(\gamma-1) \frac{T W}{\rho} \sum_{i=1}^{N_{s}} \frac{1}{W_{i}}\left(\sum_{j=1}^{N_{r}} \hat{q}_{j l}^{\sigma} \dot{r}_{i}^{j}\right)
$$

and

$$
\hat{q}_{j k}^{\eta} \equiv I_{j} \frac{\nu_{k j} \hat{\alpha}}{T}, \quad \hat{q}_{j l}^{\sigma} \equiv I_{j} \nu_{k j} \hat{\beta}, \quad 1 \leq k \leq N_{s}, \quad 1 \leq l \leq N_{s}, \quad 1 \leq j \leq N_{r} .
$$

Once canonical solutions of (22) are obtained, solutions of the original systems (14) and (19) are recovered simply by setting

$$
\boldsymbol{Z}_{, k}^{\mathrm{h}}=\frac{\alpha_{k}}{\hat{\alpha}} \hat{\boldsymbol{Z}}_{, k}^{\mathrm{h}}, \quad \text { and } \quad \boldsymbol{Z}_{, l}^{\mathrm{s}}=\frac{\beta_{l}}{\hat{\beta}} \hat{\boldsymbol{Z}}_{, l}^{\mathrm{s}} .
$$

Remark 1. In the developments above, we have assumed that the enthalpies and entropies of all species involved in the detailed mechanism are uncertain. Consequently, the indices $k$ (enthalpies) and $l$ (entropies) range from 1 to $N_{s}$. This need not always be the case, as one may alternatively focus on selected species of interest. Thus, in general the indices $k$ and $l$ may respectively range in subsets $\mathcal{K}_{\mathrm{h}} \subseteq \mathcal{I}_{\mathrm{s}}$ and $\mathcal{K}_{\mathrm{s}} \subseteq \mathcal{I}_{\mathrm{s}}$, where $\mathcal{I}_{\mathrm{s}} \equiv\left\{1, \ldots, N_{s}\right\}$.

Remark 2. It is convenient to rewrite the source terms in matrix form, i.e. letting $\mathbf{S}^{\eta}$ and $\mathbf{S}^{\sigma}$ respectively denote the matrices with components $S_{i, k}^{\eta}$ and $S_{i, l}^{\sigma}$ where $i=1, \ldots, N_{s}+1, k \in \mathcal{K}_{\mathrm{h}}$ and $l \in \mathcal{K}_{\mathrm{s}}$.

Let $\mathbf{Q}$ and $\mathbf{Q}^{\prime}$ denote the matrices respectively defined component-wise by:

$$
Q_{j k}=I_{j} \nu_{k j} \quad 1 \leq j \leq N_{r}, \quad k \in \mathcal{K}_{\mathrm{h}},
$$

and

$$
Q_{j l}^{\prime}=I_{j} \nu_{l j}, \quad 1 \leq j \leq N_{r}, \quad l \in \mathcal{K}_{\mathrm{s}} .
$$

Note that $\mathbf{Q}$ and $\mathbf{Q}^{\prime}$ are mechanism-dependent but time-independent. Also, they do not involve the reference ranges of enthalpy and entropy uncertainties, $\hat{\alpha}$ and $\hat{\beta}$.

Let $\mathbf{R}$ by the matrix defined component-wise by:




and let $\mathbf{A}$ be the "row" matrix,

$$
A_{i k}=\left\{\begin{array}{lll}
0 & 1 \leq i \leq N_{s} & k \in \mathcal{K}_{\mathrm{h}} \\
a_{k} & i=N_{s}+1 \quad k \in \mathcal{K}_{\mathrm{h}}
\end{array}\right.
$$

where

$$
a_{k} \equiv-\frac{\gamma}{\rho c_{p}} \frac{\Re}{W_{k}}\left(\sum_{j=1}^{N_{r}} \dot{\omega}_{k}^{j}\right) \quad k \in \mathcal{K}_{\mathrm{h}} .
$$

A straightforward calculation yields

and

$$
\mathbf{S}^{\eta}=\hat{\alpha} \mathbf{A}+\frac{\hat{\alpha}}{T} \mathbf{R Q}
$$

$$
\mathbf{S}^{\sigma}=-\hat{\beta} \mathbf{R} \mathbf{Q}^{\prime} .
$$

\section{Rate and thermodynamic parameter sensitivities}

The development above enables us to provide a combined treatment of local sensitivities to thermodynamic and rate parameters, in the case where the former are expressed as in section 2 above, and the latter follow the form given in [12]. Specifically, when the pre-exponents of reaction $j$ is parametrized according to

$$
A_{j}\left(\xi_{j}\right)=\mathrm{UF}_{j}^{\xi_{j}} \bar{A}_{j}, \quad j=1, \ldots, N_{r},
$$

where $\mathrm{UF}_{j}$ is the uncertainty factor associated with reaction $j$, the $\xi_{j}$ 's are canonical independent random variables uniformly distributed over $[-1,1]$, and $\bar{A}_{j}$ is the "nominal" pre-exponential of reaction $j$, the evolution of the derivative of $\boldsymbol{Z}(t)$ with respect to variations in $\xi_{j}$, denoted $\boldsymbol{Z}_{, j}^{\mathrm{r}}(t) \equiv \partial \boldsymbol{Z} / \partial \xi_{j}$, obeys to

$$
\frac{d \boldsymbol{Z}_{, j}^{\mathrm{r}}}{d t}=\mathbf{J}(t) \boldsymbol{Z}_{, j}^{\mathrm{r}}+\boldsymbol{S}_{j}^{\xi} \quad j=1, \ldots, N_{r}
$$

with initial condition $\boldsymbol{Z}_{, j}^{\mathrm{r}}(0)=0$. As before, $\mathbf{J}(t)$ is the Jacobian of the right-hand side of (1) at $\boldsymbol{Z}(t)$, whereas the source term is given by

$$
S_{i j}^{\xi}(t)= \begin{cases}\frac{1}{\rho} q_{i j}^{\xi}(t) & i=1, \ldots, N_{s} \\ -\frac{\gamma}{\rho c_{p}} \sum_{i=1}^{N_{s}} h_{i} q_{i j}^{\xi}(t)+(\gamma-1) \frac{T W}{\rho} \sum_{i=1}^{N_{s}} \frac{1}{W_{i}} q_{i j}^{\xi}(t) & i=N_{s}+1,\end{cases}
$$

where

$$
q_{i j}^{\xi}(t) \equiv \ln \left(\mathrm{UF}_{j}\right) \dot{\omega}_{i}^{j}(\boldsymbol{Z}(t)), \quad 1 \leq i \leq N_{s}+1, \quad 1 \leq j \leq N_{r} .
$$

As for the thermodynamic parameters, one can define a canonical system by introducing the scaled sensitivity vector,

$$
\hat{\boldsymbol{Z}}_{, j}^{\mathrm{r}} \equiv \frac{\boldsymbol{Z}_{, j}^{\mathrm{r}}}{\ln \left(\mathrm{UF}_{j}\right)}, \quad j=1, \ldots, N_{r},
$$

which leads to the canonical system,

$$
\frac{d \hat{\boldsymbol{Z}}_{, j}^{\mathrm{r}}}{d t}=\mathbf{J}(t) \hat{\boldsymbol{Z}}_{, j}^{\mathrm{r}}+\hat{\boldsymbol{S}}_{j}^{\xi}, \quad \hat{\boldsymbol{Z}}_{, j}^{\mathrm{r}}(0)=0, \quad j=1, \ldots, N_{r},
$$

where the vector $\hat{\boldsymbol{S}}_{j}^{\xi}$ has components $\hat{S}_{i j}^{\xi}$ given by

$$
\hat{S}_{i j}^{\xi}(t)= \begin{cases}\frac{1}{\rho} \dot{\omega}_{i}^{j}(\boldsymbol{Z}(t)) & i=1, \ldots, N_{s} \\ -\frac{\gamma}{\rho c_{p}} \sum_{i=1}^{N_{s}} h_{i} \dot{\omega}_{i}^{j}(\boldsymbol{Z}(t))+(\gamma-1) \frac{T W}{\rho} \sum_{i=1}^{N_{s}} \frac{1}{W_{i}} \dot{\omega}_{i}^{j}(\boldsymbol{Z}(t)) & i=N_{s}+1\end{cases}
$$


Remark 3. With the definitions above, one can express the TLA of the canonical sensitivities to the uncertain rates, enthalpies, and entropies, through the generic system

$$
\frac{d \hat{\boldsymbol{Z}}_{, m}^{\bullet}}{d t}=\mathbf{J}(t) \hat{\boldsymbol{Z}}_{, m}^{\bullet}+\hat{\boldsymbol{S}}_{m}^{\bullet}, \quad \hat{\boldsymbol{Z}}_{, m}^{\bullet}(0)=0, \quad m \in \mathcal{K}_{\bullet}
$$

where $\bullet \in\{\mathrm{h}, \mathrm{s}, \mathrm{r}\}$, and the source term $\hat{\boldsymbol{S}}_{m}^{\bullet}$ is $\hat{\boldsymbol{S}}_{m}^{\xi}(t)$ in $(41)$ when $\bullet=\mathrm{r}, \hat{\boldsymbol{S}}_{k}^{\eta}(t)$ in $(23)$ and $(24)$ when $\bullet=\mathrm{h}$, and $\hat{\boldsymbol{S}}_{l}^{\sigma}(t)$ as defined in $(23)$ and $(25)$ when $\bullet=$ s.

Letting $\mathbf{Z}$ denote the matrix concatenating the vectors $\hat{Z}_{, m}^{\bullet}$ and $\mathbf{T}$ the matrix concatenating the matrices $\hat{\mathbf{S}}^{\xi}$ in (41), $\mathbf{S}^{\eta}$ in (33) and $\mathbf{S}^{\sigma}$ in (34), we may express the sensitivity evolution equations as

$$
\frac{d \mathbf{Z}}{d t}=\mathbf{J}(t) \mathbf{Z}+\mathbf{T}, \quad \mathbf{Z}(0)=\mathbf{0}
$$

Because the matrices $\mathbf{S}^{\eta}$ and $\mathbf{S}^{\sigma}$ require the same terms needed to form $\hat{\mathbf{S}}^{\xi}$, when analyzing thermodynamic sensitivities the simultaneous consideration of all reaction rate sensitivities incurs a small incremental cost, and so we will generally consider $\mathcal{K}_{\mathrm{r}}=\left\{1, \ldots, N_{r}\right\}$.

Remark 4. Using an analytical transformation, an amplitude correction scheme for the rate sensitivities was developed in [12]. This amounts to multiplying the reaction rate sensitivities by a correction factor, $\Lambda$, defined according to:

$$
\Lambda=\frac{t \frac{d Z}{d t}}{\sum_{j} \hat{Z}_{, j}^{\mathrm{r}}(t) \eta_{j}}
$$

where $Z$ denotes a generic component of $\boldsymbol{Z}$. Though the amplitude correction scheme is not needed when the right-hand-side of the TLA system is accurately estimated, it was shown in [12] to dramatically reduce errors in the prediction of ignition delay time sensitivities when an approximate Jacobian is used. In the analysis below, we explore the application of this correction scheme by applying the correction to all the entries of $\mathbf{Z}$, i.e. we estimate the amplitude-corrected rate and thermodynamic sensitivities through

$$
\mathbf{Z}^{a c} \equiv \Lambda \mathbf{Z}
$$

Remark 5. With the ignition delay time, $\tau_{\text {ign }}$, defined as the time lapse needed for the mixture to rise by an increment $\Delta T_{\text {ign }}$ from its initial temperature $T_{0}$, i.e. $T\left(\tau_{\text {ign }}\right)=T_{0}+\Delta T_{\text {ign }}$, one can follow the linearized approximation approach developed in [12] to estimate the sensitivities of $\tau_{\text {ign }}$ to variations in the $\xi_{j}, \eta_{k}$ and $\sigma_{l}$. This yields

$$
\frac{\partial \tau_{\text {ign }}}{\partial \xi_{j}} \approx-\frac{\frac{\partial T}{\partial \xi_{j}}\left(\tau_{\text {ign }}\right)}{\frac{d T}{d t}\left(\tau_{\text {ign }}\right)}, \quad \frac{\partial \tau_{\text {ign }}}{\partial \eta_{k}} \approx-\frac{\frac{\partial T}{\partial \eta_{k}}\left(\tau_{\text {ign }}\right)}{\frac{d T}{d t}\left(\tau_{\text {ign }}\right)}, \quad \frac{\partial \tau_{\text {ign }}}{\partial \sigma_{l}} \approx-\frac{\frac{\partial T}{\partial \sigma_{l}}\left(\tau_{\text {ign }}\right)}{\frac{d T}{d t}\left(\tau_{\text {ign }}\right)}
$$

As in [12], we will assess the fidelity of (46) by comparing the resulting estimates with results obtained through a brute-force, finite-difference methodology based on independent (nonlinear) perturbations of $\boldsymbol{\xi}$, $\boldsymbol{\eta}$, and/or $\boldsymbol{\sigma}$. Note that, as discussed in [12], the linearization approach may be readily extended to other functionals, enabling us to define $\tau_{\text {ign }}$ based on pressure, or possibly other observables.

\section{Numerical Schemes}

The numerical solution of (1) is computed using TChem, which relies on an adaptive-step, error-controlled, stiff integration methodology to determine the time profile of state vector, $\boldsymbol{Z}(t)$. The output frequency is controlled by specifying the maximum time step, $\Delta t_{\max }$, as well as the maximum temperature difference, 
$\Delta T_{\max }$, between consecutive records. In all cases presented below, we set $\Delta T_{\max }=1 \mathrm{~K}$. This methodology results in discrete time profile of the state vector, $Z^{n}, n=0, \ldots, N$, where the superscript denotes the time level. The latter is subsequently used as input to the TLA methodology.

Specifically, we rely on a second-order Crack-Nicolson scheme to integrate (22) and (40) on the same time grid on which $\boldsymbol{Z}^{n}$ is available. Reusing the notations of Remark 3 the integration is expressed as:

$$
\hat{\boldsymbol{Z}}_{, m}^{\bullet, n+1}=\left(\mathbb{I}-\frac{1}{2} \Delta t_{n+1} \mathbf{J}^{n+1}\right)^{-1}\left[\hat{\boldsymbol{Z}}_{, m}^{\bullet, n}+\frac{1}{2} \Delta t_{n+1}\left(\mathbf{J}^{n} \hat{\boldsymbol{Z}}_{, m}^{\bullet, n}+\hat{\boldsymbol{S}}_{m}^{\bullet, n+1}+\hat{\boldsymbol{S}}_{m}^{\bullet, n}\right)\right], \quad m \in \mathcal{K}_{\bullet}
$$

where $\mathbb{I}$ is the $\left(N_{s}+1\right) \times\left(N_{s}+1\right)$ identity matrix. To estimate the Jacobian, $\mathbf{J}^{n} \equiv \mathbf{J}\left(\boldsymbol{Z}^{n}\right)$, we rely on library functions in TChem, which provides estimates using either analytical expressions or finite differences.

Note that integration of (22) and (40) yields the evolution of the corresponding sensitivities $\hat{\boldsymbol{Z}}_{m}^{\mathrm{h}}$ and $\hat{\boldsymbol{Z}}_{m}^{\mathrm{s}}$. Finally, interpolating the discrete profiles at $t=\tau_{\text {ign }}$ enables us to apply the linearized approximation (46) and consequently estimate the desired sensitivity of $\tau_{\text {ign }}$ with respect to the index $m$.

\section{Results}

In this section, we present results obtained by applying TLA schemes to three different settings. First, we consider the oxidation of hydrogen in air using a small mechanism [19]. Then, we scale up the problem considering uncertainty in a larger number of species, as they feature in the iso-octane mechanism from [20-22]. Finally, we apply TLA to estimate local sensitivities of the ignition delay time on rate and thermodynamic uncertainties belonging to rate rules and thermodynamic classes of the n-butanol mechanism from [23].

To quantitatively assess the errors in the TLA approach, we use the finite-difference results as the reference. For a vector $\mathbf{u} \in \mathbb{R}^{m}$ we denote $\|\mathbf{u}\|_{2}$ its $L_{2}$-norm, $\operatorname{RMS}(\mathbf{u}) \equiv\|\mathbf{u}\|_{2} / \sqrt{m}$ its the Root Mean Squared value, and $\|\mathbf{u}\|_{\infty}$ its infinity norm. Using these definitions, we set the normalized errors $\mathcal{E}_{2}$ and $\mathcal{E}_{\infty}$ of a TLA estimate $\mathbf{u}_{\mathrm{TLA}}$ to be

$$
\mathcal{E}_{2}=\sqrt{m} \frac{\operatorname{RMS}\left(\mathbf{u}_{\mathrm{TLA}}-\mathbf{u}_{\mathrm{FD}}\right)}{\left\|\mathbf{u}_{\mathrm{FD}}\right\|_{2}} \quad \text { and } \quad \mathcal{E}_{\infty}=\frac{\operatorname{RMS}\left(\mathbf{u}_{\mathrm{TLA}}-\mathbf{u}_{\mathrm{FD}}\right)}{\left\|\mathbf{u}_{\mathrm{FD}}\right\|_{\infty}} .
$$

In the analysis of the results, we shall denote $T_{, i}^{\mathrm{h}}\left(\operatorname{resp} . T_{, i}^{\mathrm{s}}\right.$ or $\left.T_{, i}^{\mathrm{r}}\right)$ the partial derivative $\partial T\left(\tau_{\text {ign }}\right) / \partial \eta_{i}$ (resp. $\partial T\left(\tau_{\text {ign }}\right) / \partial \sigma_{i}$ or $\left.\partial T\left(\tau_{\text {ign }}\right) / \partial \xi_{i}\right)$ for $i \in \mathcal{K}_{\mathrm{h}}\left(\right.$ resp. $\mathcal{K}_{\mathrm{s}}$ or $\left.\mathcal{K}_{\mathrm{r}}^{\prime}\right)$.

\section{1. $\mathrm{H}_{2}$ oxidation}

Here, we apply the TLA scheme to the $\mathrm{H}_{2}$ mechanism from [19], which involves $N_{s}=9$ species, and $N_{r}=$ 19 reactions. We consider hydrogen oxidation in air at stoichiometric conditions with initial temperature $T_{0}=1000 \mathrm{~K}$, and initial pressure $p_{0}=1$ bar. The computer code TChem is used to perform the computations with output frequency set to $\Delta T_{\max }=1 \mathrm{~K}$ and $\Delta t_{\max }=10^{-7} \mathrm{~s}$. These settings lead to a discrete statevector profile defined on an irregular time grid having 2649 intervals, forming a partition of the interval $\left[0, \tau_{0}\right]$, where $\tau_{0}=2.3991 \times 10^{-4} \mathrm{~s}$ is the nominal ignition delay time of the unperturbed system.

Figure 1 shows the derivatives of the ignition time with respect to the species enthalpy $\left(\partial \tau_{\mathrm{ign}} / \partial \eta_{k}\right.$, top frame) and entropy $\left(\partial \tau_{\mathrm{ign}} / \partial \sigma_{l}\right.$, bottom frame) versus their respective species index. The plots contrast the estimates obtained using finite-differences (blue circles) and the linearized approximation (orange dots). The figure displays the excellent agreement between the results obtained using finite-differences and the linearized approximation. This agreement highlights the ability of the linearized approximation (46) to provide accurate estimates of the local sensitivities of $\tau_{\text {ign }}$.

To determine the local sensitivity indices $\mathrm{S}_{k}^{\mathrm{h}}$ and $\mathrm{S}_{l}^{\mathrm{s}}$, we first exploit the TLA schemes to estimate the temperature derivatives with the species' enthalpy and entropy, $T_{, k}^{\mathrm{h}}$ and $T_{, l}^{\mathrm{s}}$. Figure 2 shows the estimates of enthalpy (top frame) and entropy (bottom frame) derivatives for all the species. Again, a close agreement between the finite-difference and TLA estimates is observed.

Figure 3 finally presents the local sensitivity indices $S_{k}^{\mathrm{h}}$ and $\mathrm{S}_{l}^{\mathrm{s}}$ computed using TLA, without amplitude correction. As for the estimates of the enthalpy and entropy derivatives shown above, an excellent agreement is seen with results obtained using the brute-force, finite-difference approach. 

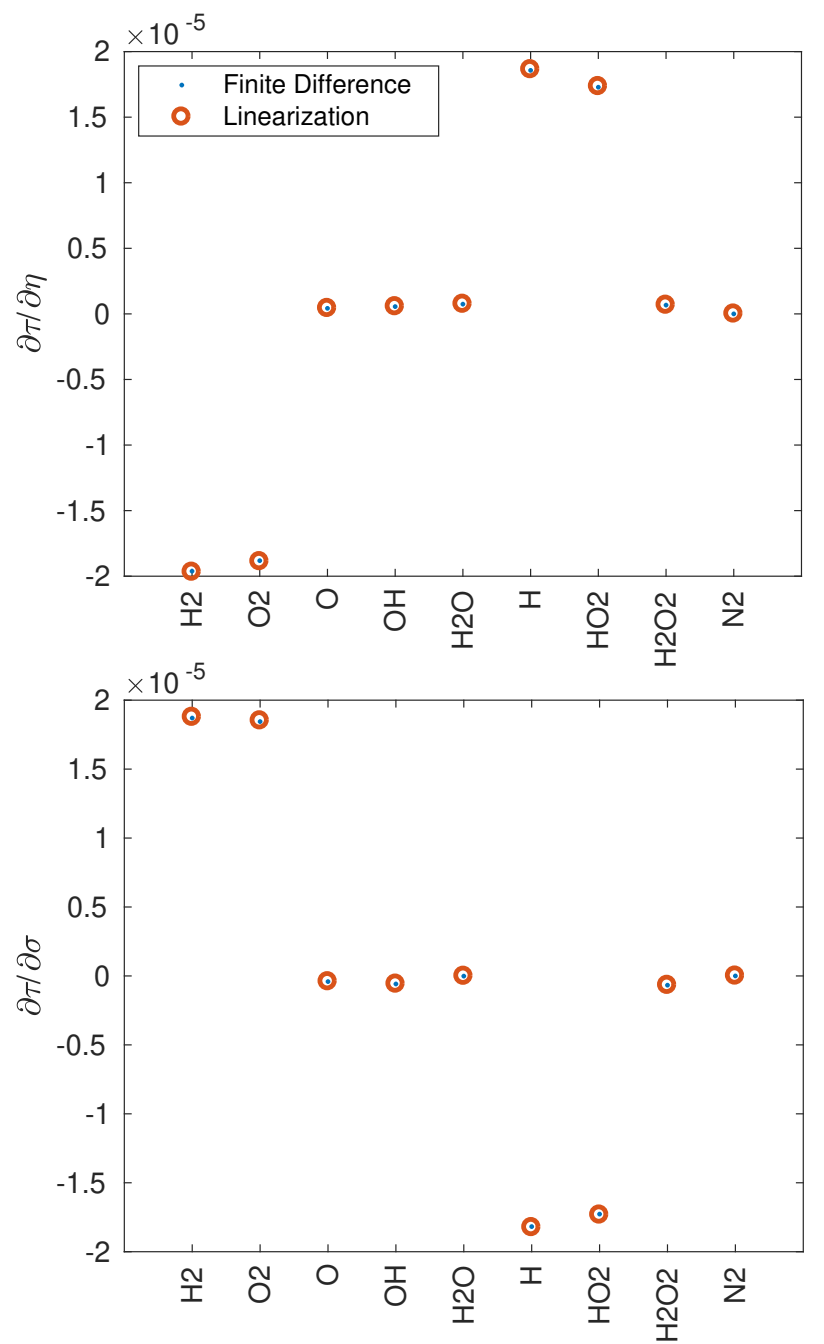

Figure 1: Derivatives of ignition time $\partial \tau_{\mathrm{ign}} / \partial \eta_{k}$ (top frame) and $\partial \tau_{\mathrm{ign}} / \partial \sigma_{l}$ (bottom frame) versus the species index for the $\mathrm{H}_{2}$ oxidation case. Plotted are estimates obtained using finite-differences (blue circles) and the linearized approximation (orange dots). The normalized errors $\mathcal{E}_{2}$ in the local sensitivity vectors are $3.4182 \times 10^{-5}$ and $3.4127 \times 10^{-5}$ for enthalpies and entropies derivatives, respectively.

Table 1 reports the normalized errors for the TLA estimates of $T_{, k}^{\mathrm{h}}$ and $T_{, l}^{\mathrm{s}}$. Noting that errors reported in Table 1 are very small, we conclude that the predictions obtained using the TLA methodology are accurate. We also note the normalized errors for $\mathrm{S}_{l}^{\mathrm{h}}$ and $\mathrm{S}_{k}^{\mathrm{s}}$ (not shown) are nearly identical to those of $T_{k}^{\mathrm{h}}$ and $T_{. l}^{\mathrm{s}}$. These similarities indicate that TLA integration errors dominate those arising from the linearized approximation.

\begin{tabular}{|c|c|c|}
\hline \multicolumn{2}{|c|}{ Enthalpy } & Entropy \\
\hline $\mathcal{E}_{2}$ & $1.4700 \mathrm{e}-04$ & $1.5240 \mathrm{e}-04$ \\
$\mathcal{E}_{\infty}$ & $9.2952 \mathrm{e}-05$ & $9.0988 \mathrm{e}-05$ \\
\hline
\end{tabular}

Table 1: Normalized errors of the TLA estimates of temperature derivatives with respect to the species' enthalpy and entropy $\left(T_{, k}^{\mathrm{h}}\right.$ and $\left.T_{l}^{\mathrm{s}}\right)$ for the $\mathrm{H}_{2}$ oxidation case. TLA results obtained without amplitude correction. 

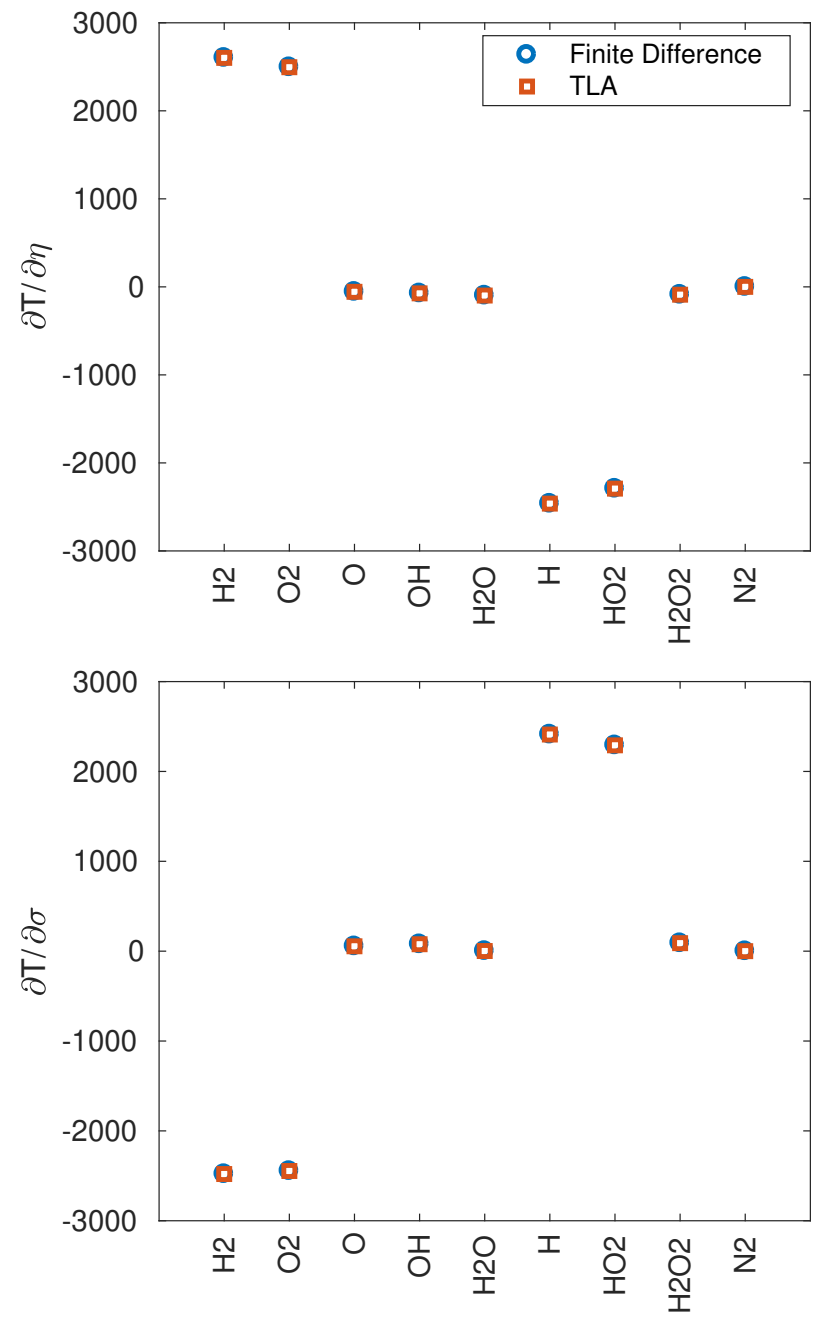

Figure 2: Derivatives $T_{k}^{\mathrm{h}}$ and $T_{l}^{\mathrm{s}}$ (bottom frame) versus the species index for $\mathrm{H}_{2}$ oxidation. Shown are results obtained using finite-differences and TLA using analytical Jacobian and time grid obtained with $\Delta t_{\max }=10^{-7} \mathrm{~s}$.

\subsection{Iso-octane oxidation}

We now consider the more elaborate iso-octane mechanism from [20-22], with $N_{s}=874$ species and $N_{r}=3796$ elementary reactions. The combustion of a stoichiometric iso-octane air mixture, reacting adiabatically at constant volume, starting from an initial temperature $T_{0}=850 \mathrm{~K}$ and an initial pressure $p_{0}=20$ bar is performed using TChem. The output frequency is controlled using $\Delta T_{\max }=1 \mathrm{~K}$, and $\Delta t_{\max }=10^{-5} \mathrm{~s}$. This yields 2569 points in the time interval $\left[0, \tau_{0}\right]$ where $\tau_{0}=0.0231 \mathrm{~s}$ is the nominal ignition delay time of the unperturbed system.

We contrast in Fig. 4 the estimates of the species enthalpy and entropy derivatives of $\tau_{\text {ign }}$ for the finitedifference approach and the linearized approximation. The finite-difference estimates' absolute values are sorted in descending order, and the dominant 20 derivatives are reported. The normalized error $\mathcal{E}_{2}$ on the TLA vector of sensitivities are $3.6979 \times 10^{-3}$ and $4.7417 \times 10^{-3}$ for enthalpies and entropies, respectively. The small relative error provides confidence in the quality of the linearized approximation of the ignition time derivatives.

In Fig. 5, we show the TLA approximation of enthalpy and entropy derivatives of the temperature $\left(T_{, k}^{\mathrm{h}}\right.$ and $\left.T_{, l}^{\mathrm{s}}\right)$ without and with amplitude correction for the same species indices shown in Fig. 4. The finite- 

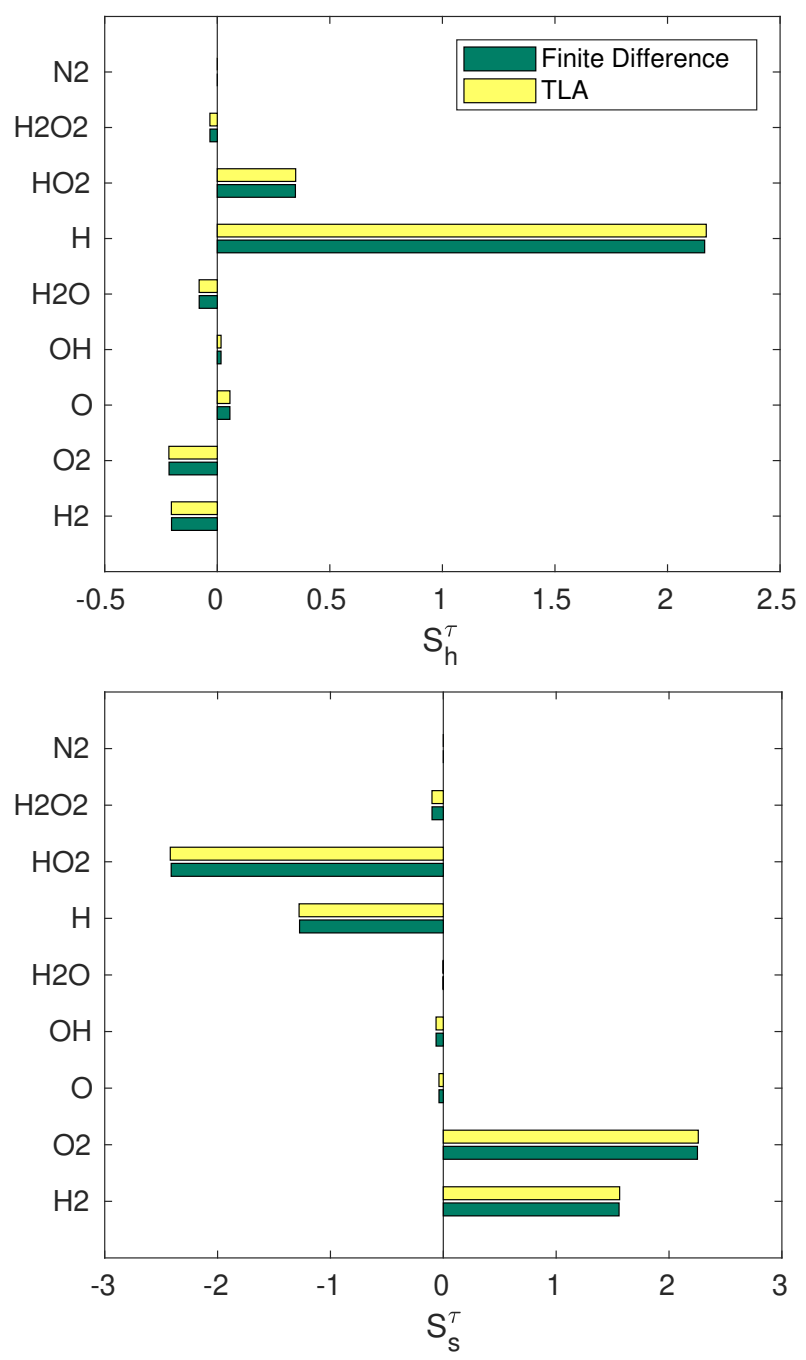

Figure 3: Sensitivity indices $S_{k}^{\text {h }}$ (top frame) and $S_{l}^{s}$ (bottom frame) associated to the enthalpy and entropy of the 9 species in the $\mathrm{H}_{2}$ oxidation mechanism. Plotted are results obtained using the finite-difference and TLA approaches, as indicated.

difference estimates are also presented for reference. We observe minor discrepancies between the uncorrected TLA results and the finite-difference estimates. However, when amplitude correction is applied, the TLA approximations of temperature derivatives are found to be in excellent agreement with the finite-difference estimates.

Figure 6 provides scatter plots of the corrected TLA estimates $T_{, j}^{\mathrm{r}}$ (rates; top frame), $T_{, k}^{\mathrm{h}}$ (enthalpies; middle frame), and $T_{, l}^{\mathrm{s}}$ (entropies; bottom frame) shown against the corresponding finite-difference estimates. Perturbations in all the pre-exponential rate parameters, species entropies, and species enthalpies are considered and depicted. As can be seen, the points in all three scatter plots fall close to the main diagonal, indicating a very close agreement between the sensitivities obtained from the TLA approximation and the finite-difference estimates for all the rate and thermodynamic parameters considered.

The TLA estimates of $\mathrm{S}_{k}^{\mathrm{h}}$ and $\mathrm{S}_{l}^{\mathrm{s}}$ are shown in Fig. 7, and are contrasted with the finite-difference estimates. Without correction, the TLA sensitivities are close to the finite-difference estimates, but applying the correction scheme effectively reduces the amplitude errors resulting from the use of a relatively coarse time grid, as the corrected TLA results are in excellent agreement with the finite-difference results.

A quantitative assessment of TLA approximation errors was again conducted using (48), namely to 


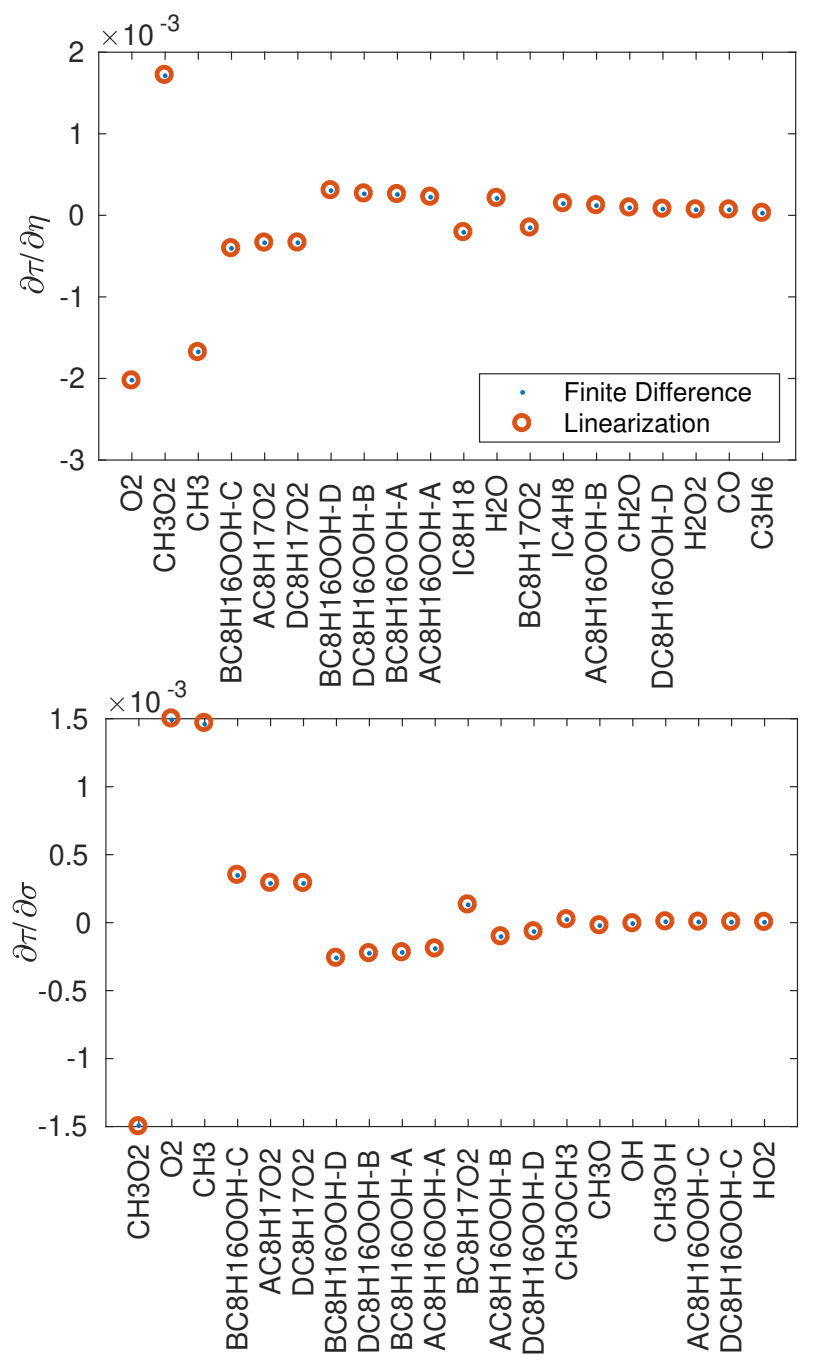

Figure 4: Derivatives of the ignition time with respect to the species enthapy ( $\partial \tau_{\mathrm{ign}} / \partial \eta_{k}$, top frame) and entropy ( $\partial \tau / \partial \sigma_{l}$, bottom frame) for iso-octane oxidation. Plotted are estimates obtained using finite-differences (blue dots) and the linearized approximation (orange circles). The reaction indices shown correspond to the largest absolute values of the finite-difference estimates of derivatives.

determine the $N_{s}$-dimensional sensitivities $T_{, k}^{\mathrm{h}}, \mathrm{S}_{k}^{\mathrm{h}}, T_{l,}^{\mathrm{s}}$ and $\mathrm{S}_{l}^{\mathrm{s}}$. As in the case of $\mathrm{H}_{2}$, the results for $T_{, k}^{\mathrm{h}}$ and $\mathrm{S}_{k}^{\mathrm{h}}$, and $T_{, l}^{\mathrm{s}}$ and $\mathrm{S}_{l}^{\mathrm{s}}$ are nearly identical and the results are only are reported for the temperature in Table 2. The normalized errors for TLA approximation without and with amplitude correction are found to be small for both enthalpy and entropy derivatives. Thus, the TLA methodology is deemed to be effective for this detailed mechanism involving hundreds of species and thousands of reactions.

\begin{tabular}{|c|cc|cc|}
\hline \multicolumn{3}{|c|}{ Enthalpy } & \multicolumn{2}{c|}{ Entropy } \\
\hline \multicolumn{2}{|c|}{ No Correction } & Correction & No Correction & Correction \\
\hline $\mathcal{E}_{2}$ & $4.9563 \mathrm{e}-02$ & $2.2188 \mathrm{e}-03$ & $4.6899 \mathrm{e}-02$ & $2.8177 \mathrm{e}-03$ \\
$\mathcal{E}_{\infty}$ & $2.7079 \mathrm{e}-03$ & $1.2123 \mathrm{e}-04$ & $2.8334 \mathrm{e}-03$ & $1.7023 \mathrm{e}-04$ \\
\hline
\end{tabular}

Table 2: Normalized error for $T_{, k}^{\mathrm{h}}$ and $T_{, l}^{\mathrm{s}}$ for the iso-octane oxidation case. Results are shown for TLA using the analytical Jacobian, with and without amplitude correction. 

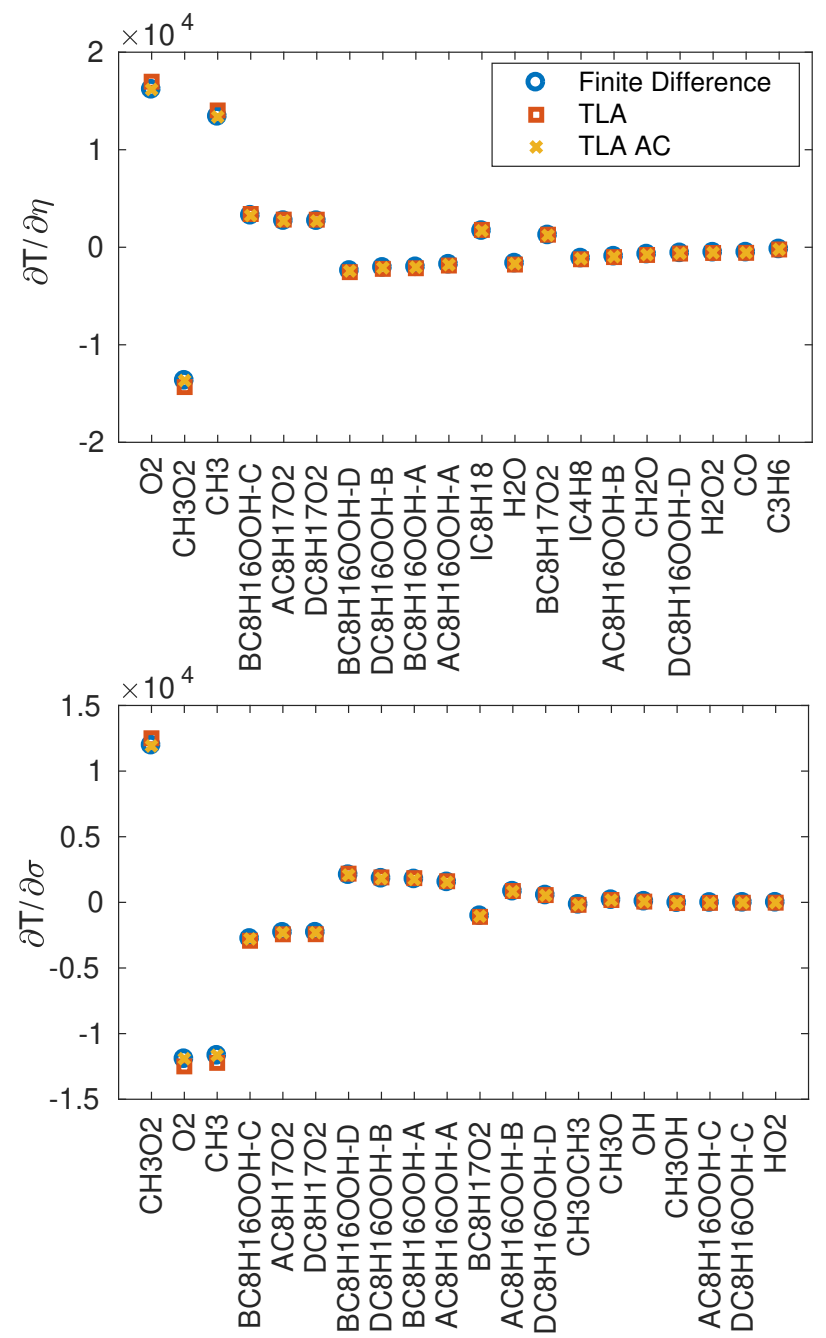

Figure 5: Estimates of the enthalpy and entropy derivatives of the temperature $\left(T_{. k}^{\mathrm{h}}\right.$ and $\left.T_{, l}^{\mathrm{s}}\right)$ for the species index shown in Fig. 4 and the iso-octane oxidation mechanism. Shown are results obtained using finite-differences and TLA using analytical Jacobian.

\section{3. $n$-butanol oxidation}

Lastly, we focus on the oxidation of a stoichiometric n-butanol/air mixture with initial temperature, $T_{0}=900 \mathrm{~K}$, and initial pressure, $p_{0}=40$ bar. The n-butanol mechanism of Sarathy et al. [23] comprising $N_{s}=687$ species and $N_{r}=3435$ reactions is used for this purpose. The constant volume simulation is performed using TChem with $\Delta T_{\max }=1 \mathrm{~K}$, and $\Delta t_{\max }=10^{-6} \mathrm{~s}$. With the selected output control parameters, the discrete states are recorded at the nodes of an irregular time grid having 17766 intervals that form an irregular partition of the interval $\left[0, \tau_{0}\right]$, where $\tau_{0}=1.769 \times 10^{-3} \mathrm{~s}$ is the ignition delay time of the unperturbed mixture.

In the present analysis of sensitivities to thermodynamic parameters, we focus exclusively on $N_{s}^{\prime}=54$ species involved in the thermodynamic classes outlined in [8] and consisting of the fuel and fuel radicals, as detailed in the Supplementary Material. In other words, the cardinality of the sets $\mathcal{K}_{\mathrm{h}}$ and $\mathcal{K}_{\mathrm{s}}$ are $\left|\mathcal{K}_{\mathrm{h}}\right|=\left|\mathcal{K}_{\mathrm{s}}\right|=54$. This restriction limits the number of independent computations in the finite-difference approach to 108 for one-sided differences and to 216 in the case of centered differences. In applying the TLA, we explored the use of both the analytical and numerical Jacobian libraries. This specific study is motivated because, in the presently used release of TChem, the analytical Jacobian expressions for PLOG reactions 

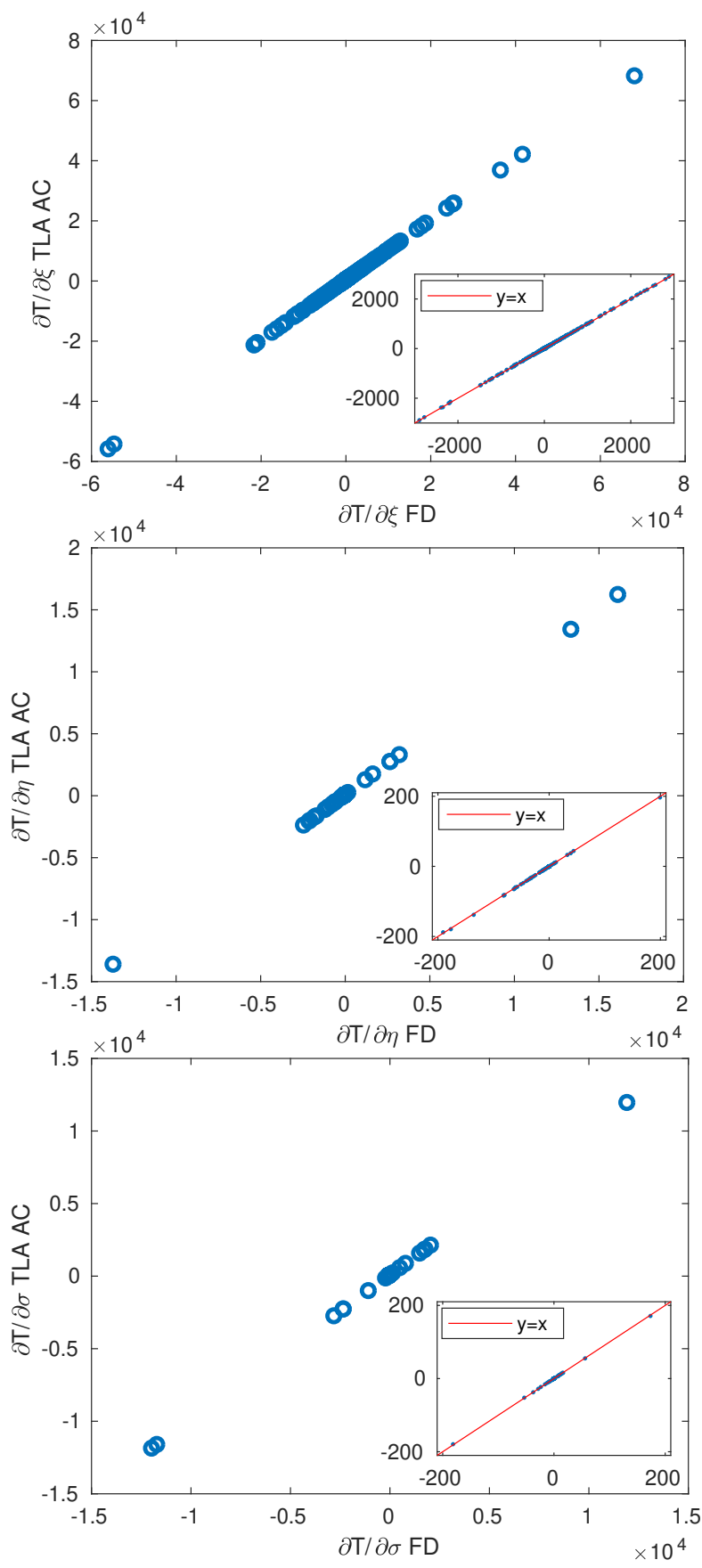

Figure 6: Scatter plots of the $T_{, j}^{\mathrm{r}}$ (top frame) $T_{, k}^{\mathrm{h}}$ (middle frame) and $T_{, l}^{\mathrm{s}}$ (bottom frame) for the iso-octane case. The TLA results ( $y$ axis) are plotted against the corresponding finite-difference estimates ( $x$ axis). The insets provide enlarged views of values around 0 . The corrected TLA results obtained using the analytical Jacobian are used.

are inexact as they ignore the pressure dependence of Arrhenius coefficients. This test case enables us to investigate whether the amplitude correction scheme provides effective means for overcoming the resulting errors, as previously observed in [12] for rate sensitivities.

Figure 8 shows the leading sensitivities $\partial \tau_{\mathrm{ign}} / \partial \eta_{k}$ (top frame), and $\partial \tau_{\mathrm{ign}} / \partial \sigma_{l}$ (bottom frame), and contrasts 

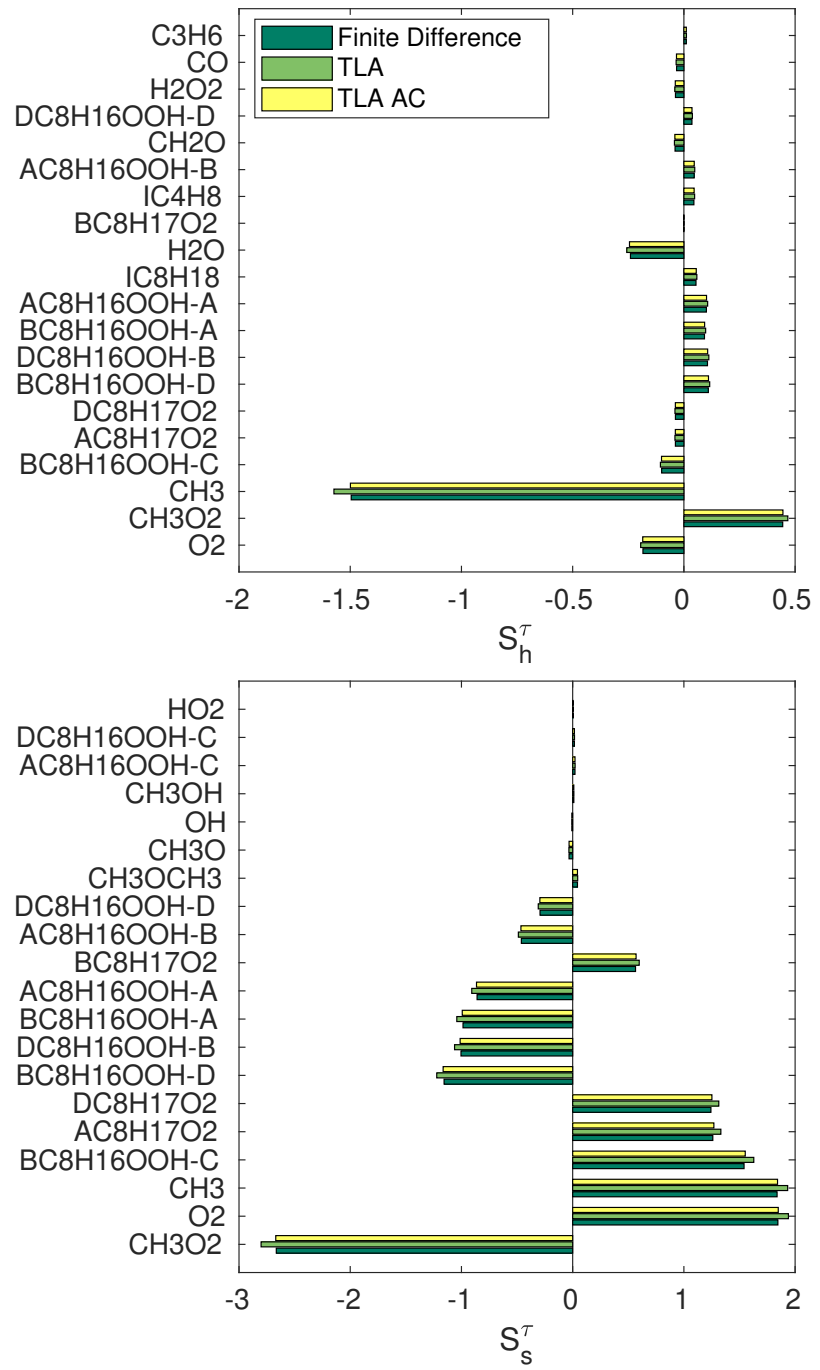

Figure 7: Sensitivity indices, $S_{k}^{\text {h }}$ (top frame) and $S_{l}^{s}$ (bottom frame), of the dominant species, arranged from bottom to top, in the iso-octane oxidation case. Plotted are results obtained using finite-differences, and TLA approximations shown in Fig. 5.

the finite-difference (blue dots) and linearized approximation (orange circle) estimates. The normalized error $\mathcal{E}_{2}$ on the vector of 58 local sensitivities is $7.7403 \times 10^{-3}$, for both enthalpies and entropies. We thus conclude that, as in the previous examples, there is an excellent agreement between the linearized approximation and the brute-force approaches.

Figure 9 depicts estimates of derivatives $T_{, k}^{\mathrm{h}}$ (top frame) and $T_{, l}^{\mathrm{s}}$ (bottom frame) obtained using finitedifferences, and TLA approximations based on the analytical Jacobian, the analytical Jacobian with amplitude correction, and the numerical Jacobian. The TLA results obtained using the numerical Jacobian (without correction) are clearly in excellent agreement with the finite-difference estimates. On the other hand, significant amplitude errors are observed when the analytical Jacobian is used. The larger errors are not surprising because, as previously noted, the analytical Jacobian expressions are presently only approximate. Note, however, that when the amplitude correction is applied to the TLA results obtained with the analytical Jacobian, the results are once again consistent with finite-difference predictions. Thus, the amplitude correction scheme effectively addresses errors resulting from the use of approximate Jacobian expressions, similar to what was observed in [12] for reaction rate sensitivities. 

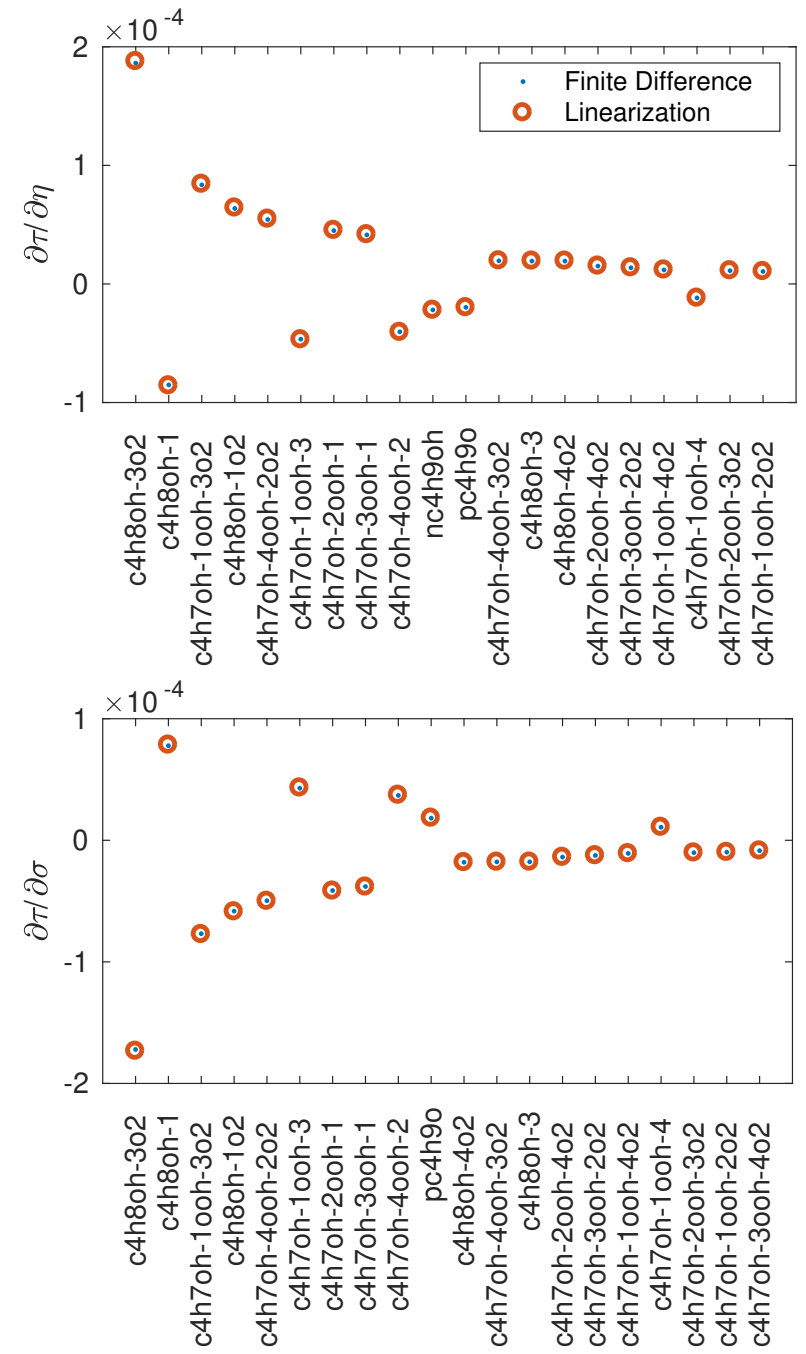

Figure 8: Dominant ignition time derivatives $\partial \tau / \partial \eta_{k}$ (top frame) and $\partial \tau / \partial \sigma_{l}$ (bottom frame) for n-butanol oxidation. Plotted are estimates obtained using finite-differences and the linearized approximation, as indicated.

The leading sensitivity indices to enthalpies and entropies of selected species, respectively $\mathrm{S}_{l}^{\mathrm{h}}$ and $\mathrm{S}_{k}^{\mathrm{s}}$ are shown in Fig. 10. In particular, the figure provides two frames that contrast TLA and finite-difference predictions. Briefly, an inspection of the results leads to similar conclusions as those drawn for the analysis of the temperature sensitivities given in Fig. 9.

To provide a quantitative assessment of the differences between TLA and finite-difference results, we estimate the normalized errors of the vector of temperature sensitivities associated with the species involved in the thermodynamic classes. Table 3 provides the normalized errors $\mathcal{E}_{2}$ and $\mathcal{E}_{\infty}$ computed for the vector of $T_{l}^{\mathrm{h}}$ and $T_{, k}^{\mathrm{s}}$. Similar to the $\mathrm{H}_{2}$ and iso-octane cases, the results show relatively small errors. Inparticular, in the present example smaller errors arise when using the numerical Jacobian without correction.

So far, we have focused exclusively on the computation of the canonical sensitivities, using fixed values of the uncertainty ranges for species enthalpies and entropies, $\bar{\alpha}=1000, \bar{\beta}=1$, and fixed value of the uncertainty factor for the rate expressions, UF $=\exp (1)$. We now turn our attention to the analysis of the temperature sensitivities associated with the actual ranges, $\mathrm{UF}_{j}, \alpha_{k}$, and $\beta_{l}$, as given in Supplementary Material for the reactions appearing in the rate rules of the n-butanol mechanism, and for the thermodynamic classes. Specifically, we rely on (39) and (27) to reverse the canonical scalings. 



Figure 9: Dominant temperature derivatives $T_{, k}^{\mathrm{h}}$ (top frame) and $T_{, l}^{\mathrm{s}}$ (bottom frame) for n-butanol oxidation. Shown are results obtained using finite-differences, and TLA approximations as indicated.

\begin{tabular}{|c|c|cc|cc|}
\hline \multicolumn{5}{|c|}{ Anal Jac } & \multicolumn{2}{c|}{ Num Jac } \\
\hline \multicolumn{2}{|c|}{ No Correction } & Correction & No Correction & Correction \\
\hline enthalpy & $\mathcal{E}_{2}$ & 0.4427 & $9.3833 \mathrm{e}-03$ & $2.3022 \mathrm{e}-03$ & $6.8683 \mathrm{e}-03$ \\
& $\mathcal{E}_{\infty}$ & $6.0251 \mathrm{e}-02$ & $1.277 \mathrm{e}-03$ & $4.3458 \mathrm{e}-04$ & $1.2877 \mathrm{e}-03$ \\
\hline entropy & $\mathcal{E}_{2}$ & 0.4428 & $9.4208 \mathrm{e}-03$ & $2.3050 \mathrm{e}-03$ & $6.8712 \mathrm{e}-03$ \\
& $\mathcal{E}_{\infty}$ & $6.0253 \mathrm{e}-02$ & $1.2820 \mathrm{e}-03$ & $3.1368 \mathrm{e}-04$ & $9.3505 \mathrm{e}-04$ \\
\hline
\end{tabular}

Table 3: Normalized errors $\mathcal{E}_{2}$ and $\mathcal{E}_{\infty}$ for $T_{, k}^{\mathrm{h}}$ and $T_{, l}^{\mathrm{s}}$ in the n-butanol oxidation case. Error estimates obtained before and after amplitude correction are reported, using TLA results with the analytical or numerical Jacobian.

Figure 11 depicts the results for the local temperature sensitivities to uncertainties in the reaction rates appearing in the rate rules $\left(T_{, j}^{\mathrm{r}}\right)$, and to the enthalpies $\left(T_{, k}^{\mathrm{h}}\right)$ and entropies $\left(T_{, l}^{\mathrm{s}}\right)$ of species appearing in the thermodynamic classes. The results indicate that the sensitivities to rates span a larger interval of values, followed by enthalpy and entropy sensitivities. Overall, however, the three ranges of temperature 

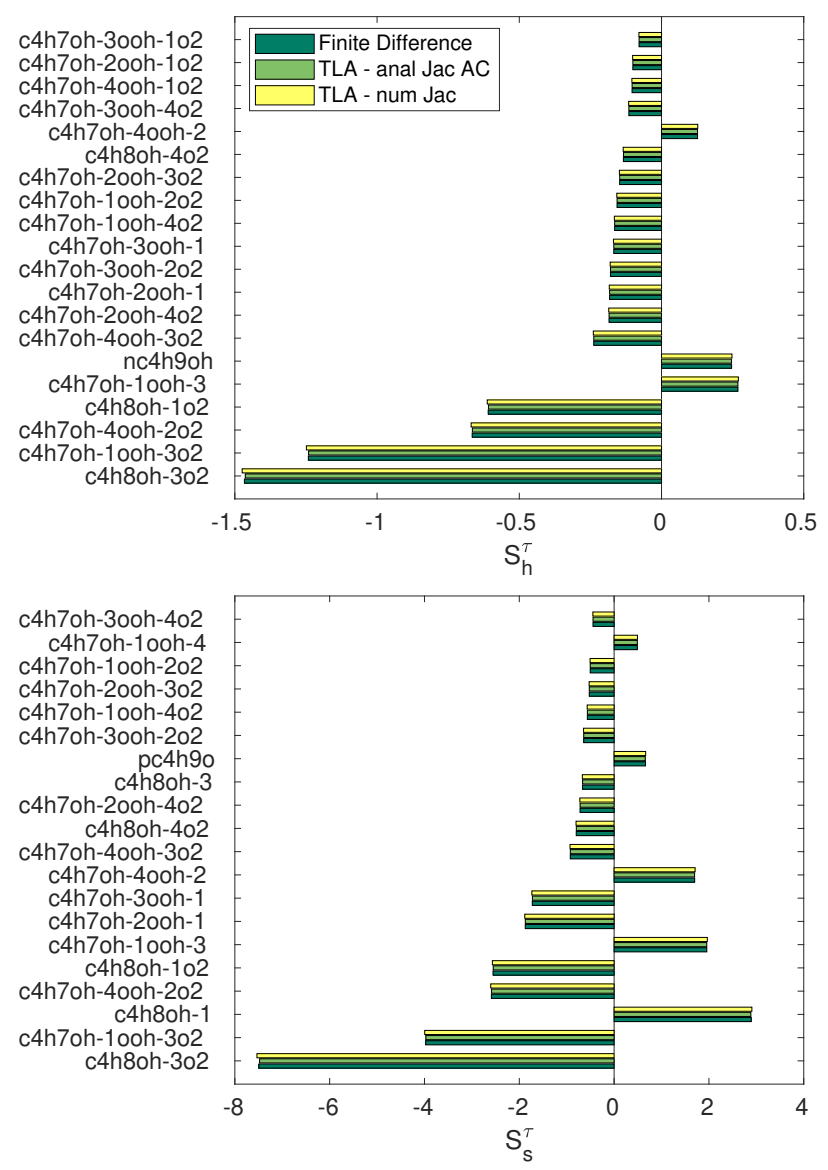

Figure 10: Leading sensitivity indices, $S_{l}^{\text {h }}$ (top frame) and $S_{k}^{\text {s }}$ (bottom frame), for individual reactions in the n-butanol oxidation case. Plotted are results obtained using finite-differences, and TLA approximations as indicated.

sensitivities are comparable.

A close-up view of the leading sensitivities is shown in Fig. 12, which shows sensitivities having absolute values greater than $10^{4} \mathrm{~K}$. As can be seen, the highest sensitivity of temperature (and consequently $\tau_{\text {ign }}$ ) occurs for an $\mathrm{H}$-atom abstraction reaction from the fuel. However, uncertainties in fuel radicals enthalpies, namely $\mathrm{C} 4 \mathrm{H} 8 \mathrm{OH}-3 \mathrm{O} 2$, C4H7OH-1OOH-3O2, $\mathrm{C} 4 \mathrm{H} 8 \mathrm{OH}-1 \mathrm{O} 2$, and $\mathrm{C} 4 \mathrm{H} 7 \mathrm{OH}-4 \mathrm{OOH}-2 \mathrm{O} 2$, also feature in the leading set. Their presence suggests a simultaneous treatment of reaction rate and thermodynamic parameter sensitivities, a goal which we will pursue in future work.

\subsection{Performance}

We conclude by briefly examining the tangent linear approximation's performance enhancement by contrasting the CPU cost incurred by TLA with the CPU cost incurred by a first-order, finite-difference methodology. In the latter case, the rates, enthalpies, and entropies are individually perturbed, and an independent simulation is performed for each perturbation. If we use the cost of a forward simulation to normalize the CPU cost, the computational cost of all sensitivities is $N_{r}+2 \times N_{s}$ when perturbations in all reaction rates, species enthalpies, and species entropies are considered, as is the case in the $\mathrm{H} 2$ and iso-octane oxidation examples. On the other hand, the computational cost is $N_{r}^{\prime}+2 \times N_{s}^{\prime}$ for n-butanol because the analysis is restricted to reactions featuring the rate rules and species appearing in the thermodynamic classes.

Table 4 contrasts the computational cost of TLA and finite-difference approaches for all three examples considered. In all cases, the same normalizing factor is used. As can be seen, in the case of $\mathrm{H}_{2}$ and nbutanol, the CPU cost incurred by TLA is approximately an order of magnitude smaller than that required 


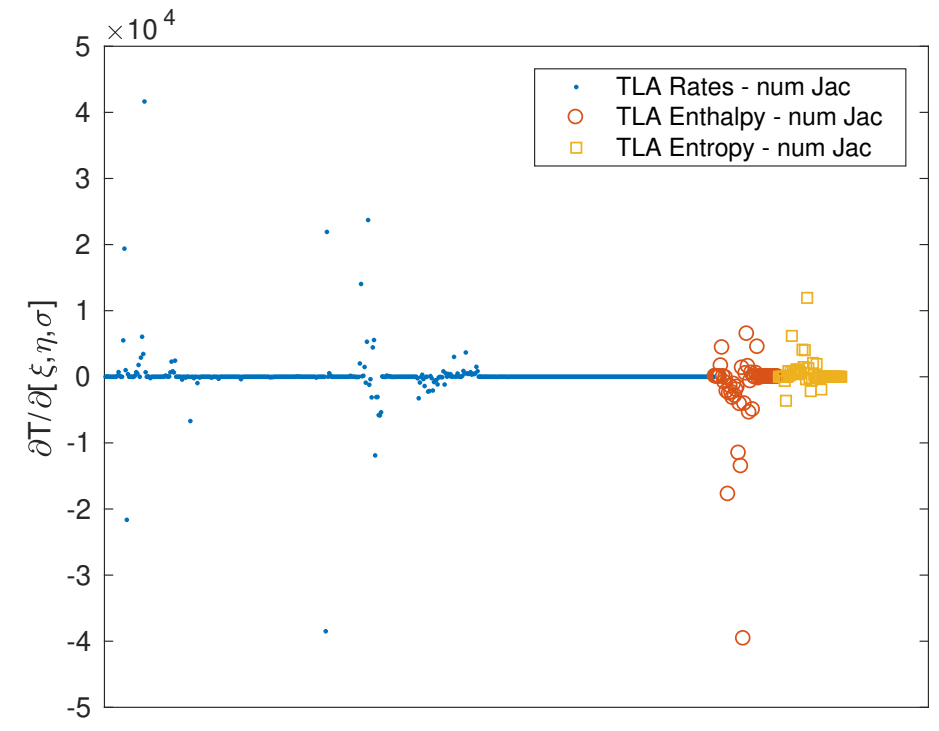

Figure 11: Scatter plots of the unscaled sensitivities $T_{, j}^{\mathrm{r}}$ (blue dots; rates), $T_{, k}^{\mathrm{h}}$ (red circles; enthalpies) and $T_{, l}^{\mathrm{s}}$ (orange squares; entropies) for the n-butanol case.

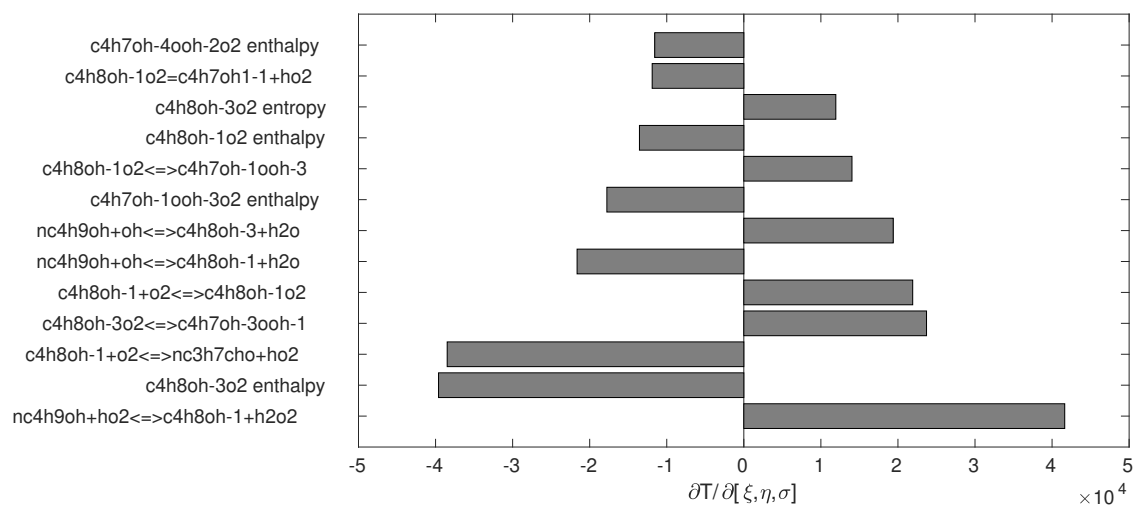

Figure 12: Leading temperature sensitivities for the n-butanol case.

by the brute force approach. For the iso-octane example, with perturbations in all parameters considered, TLA's speed-up is about three orders of magnitude. This speed-up provides effective means for leveraging local sensitivities in uncertainty quantification studies aiming to build surrogate representations and leverage these representations in global sensitivity analysis and parameter calibration.

\begin{tabular}{|c|c|c|c|}
\hline \multicolumn{2}{|c|}{$\mathrm{H}_{2}$} & n-butanol & iso-octane \\
\hline$\Delta t_{\max }$ & $10^{-7}$ & $10^{-6}$ & $10^{-5}$ \\
\hline FD & 37 & 626 & 5544 \\
Anal Jac & 4.33 & 11.78 & 6.23 \\
Num Jac & 4.17 & 11.07 & 5.71 \\
\hline
\end{tabular}

Table 4: Computational cost of various approaches, normalized by the CPU time of a single forward simulation. Reported are the CPU costs of the finite-difference (FD) approach, of the TLA using analytical and numerical Jacobians. 


\section{Conclusions}

The tangent linear approximation presented in [12] was extended to estimate the local sensitivity of the ignition delay time with respect to uncertainty in thermodynamic parameters, namely, species enthalpies and entropies. Attention is focused on a gas mixture reacting adiabatically at constant volume. The variability in the enthalpies and entropies of species is introduced by perturbing selected coefficients in the NASA polynomials so that the corresponding uncertainty ranges are temperature independent. These coefficients are parametrized using independent canonical random variables, uniformly distributed over [-1,1].

Following the methodology developed in [12], a framework was proposed that combines two key ingredients. The first ingredient consists of deriving a canonical tangent linear system governing the state vector's sensitivities on the germ's components. The second ingredient consists of adopting a linearized approximation of the functional defining the ignition delay time, thus relating the latter to the state vector's sensitivities.

Three different settings were considered to evaluate the proposed TLA, focusing on the combustion of hydrogen, n-butanol, and iso-octane in air at stoichiometric conditions and intermediate pressures and temperatures. In each case, results obtained from the TLA approach are compared against results obtaining using the finite-difference approach, based on performing independent simulations for individual perturbations in enthalpies and entropies.

In all cases considered, the linearized approximation of the ignition delay time's local sensitivity was found to be in excellent agreement with finite difference estimates. Also, the TLA results were in good agreement with finite-difference predictions. A performance analysis was finally conducted considering uncertainties in reaction rates and thermodynamic parameters simultaneously. The analysis showed that TLA is computationally competitive compared with the brute-force approach, even when the reaction mechanism involves a small number of species and a moderate number of reactions. In the case of iso-octane oxidation using a detailed mechanism with hundreds of species and thousands of elementary reactions, TLA's speed-up factors were about three orders of magnitude.

In future work, we plan to leverage TLA's capabilities, in conjunction with coordinate transform and active subspace methodologies, to examine the global impact of uncertainties in reaction rates and thermodynamic parameters in high-dimensional settings.

\section{Acknowledgments}

The research reported in this publication was supported by King Abdullah University of Science and Technology (KAUST).

\section{Appendix A.}

This appendix provides brief details, largely adapted from [24], regarding how the energy equation is derived. To arrive at the form given in (1), one starts from the conservation of the internal energy,

$$
\frac{d(\rho u)}{d t}=0,
$$

where $u \equiv \sum_{i} u_{i} Y_{i}=\sum_{i} h_{i} Y_{i}-R T$ is the internal energy of the system, and $u_{i}$ is the internal energy of species $i$. Because $\rho$ is constant, we have:

$$
\frac{d(\rho u)}{d t}=\rho \sum_{i=1}^{N_{s}} Y_{i} c_{p, i} \frac{d T}{d t}+\rho \sum_{i=1}^{N_{s}} h_{i} \frac{d Y_{i}}{d t}-\frac{d p}{d t}
$$

where $p=\rho R T$ is the thermodynamic pressure, and

$$
R \equiv \Re \sum_{i=1}^{N_{s}} \frac{Y_{i}}{W_{i}} .
$$


Combining (A.1-A.3), we obtain:

$$
\frac{d T}{d t}=-\frac{1}{\rho c_{p}} \sum_{i=1}^{N_{s}} h_{i} \dot{\omega}_{i}+\frac{1}{\rho c_{p}} \frac{d p}{d t}
$$

where $c_{p}=\sum_{i=1}^{N_{s}} Y_{i} c_{p, i}$ is the heat capacity of the mixture. Differentiating the equation of state,

$$
p=\rho \Re\left[\sum_{i=1}^{N_{s}} \frac{Y_{i}}{W_{i}}\right] T,
$$

and using the fact that $\rho$ is constant, we have:

$$
\frac{d p}{d t}=W R T \sum_{i=1}^{N_{s}} \frac{\dot{\omega}_{i}}{W_{i}}+\rho R \frac{d T}{d t},
$$

where $W$ is given by (2). Substituting (A.6) into (A.4), we get:

$$
\frac{d T}{d t}=-\frac{1}{\rho c_{p}} \sum_{i=1}^{N_{s}} h_{i} \dot{\omega}_{i}+\frac{1}{\rho c_{p}}\left[W R T \sum_{i=1}^{N_{s}} \frac{\dot{\omega}_{i}}{W_{i}}+\rho R \frac{d T}{d t}\right],
$$

which readily yields:

$$
\left[1-\frac{R}{c_{p}}\right] \frac{d T}{d t}=-\frac{1}{\rho c_{p}} \sum_{i=1}^{N_{s}} h_{i} \dot{\omega}_{i}+\frac{R}{c_{p}} \frac{T W}{\rho} \sum_{i=1}^{N_{s}} \frac{\dot{\omega}_{i}}{W_{i}} .
$$

Substituting the relation $R=c_{p}(\gamma-1) / \gamma$ into (A.7), expressing $\dot{\omega}_{i}$ in terms of the forward and reverse rates, $\dot{\omega}_{i}=\sum_{j=1}^{N_{r}}\left[\dot{f}_{i}^{j}+\dot{r}_{i}^{j}\right]$, and rearranging, we finally arrive at the form of the energy equation given in (1).

\section{References}

[1] J. Bromly, F. Barnes, S. Muris, X. You, B. Haynes, Kinetic and thermodynamic sensitivity analysis of the NO-sensitised oxidation of methane, Combust. Sci. Technol. 115 (1996) 259-296.

[2] T. Turányi, L. Zalotai, S. Dóbé, T. Bérces, Effect of the uncertainty of kinetic and thermodynamic data on methane flame simulation results, Phys. Chem. Chem. Phys. 4 (2002) 2568-2578.

[3] I. G. Zsély, J. Zádor, T. Turányi, Uncertainty analysis of updated hydrogen and carbon monoxide oxidation mechanisms, Proc. Combust. Inst. 30 (2005) 1273-1281.

[4] I. G. Zsély, J. Zádor, T. Turányi, Uncertainty analysis of NO production during methane combustion, Int. J. Chem. Kinet. 40 (2008) 754-768

[5] F. vom Lehn, L. Cai, H. Pitsch, Sensitivity analysis, uncertainty quantification, and optimization for thermochemical properties in chemical kinetic combustion models, Proc. Combust. Inst. 37 (2019) 771-779.

[6] F. vom Lehn, L. Cai, H. Pitsch, Impact of thermochemistry on optimized kinetic model predictions: Auto-ignition of diethyl ether, Combust. Flame 210 (2019) 454-466.

[7] F. vom Lehn, L. Cai, H. Pitsch, Investigating the impacts of thermochemical group additivity values on kinetic model predictions through sensitivity and uncertainty analyses, Combust. Flame 213 (2020) 394-408.

[8] M. Hantouche, S. M. Sarathy, O. M. Knio, Global sensitivity analysis of n-butanol ignition delay times to thermodynamics class and rate rule parameters, Combust. Flame 222 (2020) 355-369.

[9] W. Ji, Z. Ren, C. Law, Evolution of sensitivity directions during autoignition, Proc. Combust. Inst. 37 (2019) $807-815$.

[10] M. Lemke, L. Cai, J. Reiss, H. Pitsch, J. Sesterhenn, Adjoint-based sensitivity analysis of quantities of interest of complex combustion models, Combust. Theory Model. 23 (2019) 180-196.

[11] V. Gururajan, F. Egolfopoulos, Direct sensitivity analysis for ignition delay times, Combust. Flame 209 (2019) $478-480$.

[12] S. Almohammadi, M. Hantouche, O. L. Maitre, O. Knio, A tangent linear approximation of the ignition delay time. I: Sensitivity to rate parameters, Combust. Flame 230 (2021) 111426.

[13] A. E. Lutz, R. J. Kee, J. A. Miller, SENKIN: A FORTRAN program for predicting homogeneous gas phase chemical kinetics with sensitivity analysis, Technical Report, Sandia National Labs., Livermore, CA (USA), 1988.

[14] T. Turányi, Sensitivity analysis of complex kinetic systems. Tools and applications, J. Math. Chem. 5 (1990) 203-248.

[15] T. Turányi, Applications of sensitivity analysis to combustion chemistry, Reliab. Eng. Syst. Safe. 57 (1997) 41-48.

[16] R. J. Kee, F. M. Rupley, J. A. Miller, The chemkin thermodynamic data base, https://www.osti.gov/biblio/7073290, 1990.

[17] C. Safta, H. Najm, O. Knio, TChem - A Software Toolkit for the Analysis of Complex Kinetic Models, Technical Report Report No. SAND2011-3282, Sandia National Laboratories, Livermore, CA, USA, 2011. 
[18] B. J. McBride, S. Gordon, M. A. Reno, Coefficients for Calculating Thermodynamic and Transport Properties of Individual Species, Technical Report NASA TM-4513, NASA, 1993.

[19] R. Yetter, F. Dryer, H. Rabitz, A comprehensive reaction mechanism for carbon monoxide/hydrogen/oxygen kinetics, Combust. Sci. Technol. 79 (1991) 97-128.

[20] M. Mehl, H. Curran, W. Pitz, C. Westbrook, iso-octane, version 3, https://combustion.llnl.gov/mechanisms/alkanes/isooctane-version-3, 2009

[21] M. Mehl, H. Curran, W. Pitz, C. Westbrook, in: 4th European Combustion Meeting, Vienna, Austria, April 14-17.

[22] M. Mehl, W. Pitz, M. Sjöberg, J. E. Dec, in: SAE Technical Paper 2009-01-1806.

[23] S. M. Sarathy, P. Oßwald, N. Hansen, K. Kohse-Höinghaus, Alcohol combustion chemistry, Prog. Energy Combust. Sci. 44 (2014) 40-102.

[24] C. Safta, Constant volume ignition, private communication, 2019. 